\title{
The design and performance of an ion trap storage-reflectron time-of-flight mass spectrometer
}

\author{
Benjamin M. Chien, Steven M. Michael, David M. Lubman* \\ Department of Chemistry, The University of Michigan, Ann Arbor, MI 48109, USA
}

(Received 1 March 1993; accepted 8 June 1993)

\begin{abstract}
The design and performance of an ion trap storage-reflectron time-of-flight mass spectrometer (IT-RETOF MS) combination have been explored for detection of ions generated from both internal and external ionization sources. Ions were generated inside the trap using laser-induced resonance enhanced multiphoton ionization at $266 \mathrm{~nm}$, while ions were generated external to the trap using either an atmospheric pressurc or low pressure d.c. plasma source or an electrospray ionization source. It is demonstrated herein that the ion trap provides an effective means of storing ions from $10 \mu \mathrm{s}$ up to $10 \mathrm{~s}$ prior to mass analysis via pulsed d.c. extraction into the RETOF MS device. In addition, it is shown that the storage capabilities of the device provide enhanced resolution and sensitivity as the storage time is increased. A resolution of nearly 2100 at $\mathrm{m} / z 93$ is demonstrated using external injection from the atmospheric pressure d.c. plasma source when a storage time of $9 \mathrm{~s}$ is used before ejection. Further, a resolution of $\approx 3300 \mathrm{at} \mathrm{m} / \mathrm{z} 1000$ is demonstrated using external injection from an electrospray ionization source into the trap with a storage time of $931 \mathrm{~ms}$. The IT-RETOF storage capabilities are shown to provide the potential for nearly $100 \%$ duty cycle in converting a continuous ion beam into a pulsed source for TOF. The detection limit of the device is demonstrated with liquid injection techniques for a typical sample and found to be in the low femtomole range. In addition, the r.f. voltage was shown to be an effective means of eliminating low mass background ions from the trap and, thus, from the TOF mass spectra obtained.
\end{abstract}

Key words: Ion trap storage; Reflectron; Resonance enhanced multiphoton ionization; Electrospray ionization; Atmospheric pressure ionization

\section{Introduction}

In this article we present the design and performance of a novel ion trap storage-reflectron timeof-flight (IT-RETOF MS) mass spectrometer combination. This hybrid instrument provides capabilities which combine some of the best features of the ion trap and time-of-flight devices. The time-offlight mass spectrometer has recently become widely used because of several distinct advantages [1-17]. These advantages include the fact that TOF devices can rapidly measure an entire mass spec-

*Corresponding author. trum on every ionization pulse. This property of time-of-flight is particularly important in experiments where the ionization source has a low duty cycle as in laser ionization $[3,5,6,8]$ or in experiments where rapid monitoring of transient species may be required. Other advantages include the potentially high resolution that can be achieved with TOF devices. A number of methods including supersonic jet cooling $[5,6,8]$ and an ion reflecting mirror, i.e. a reflectron [7-11], have been used to achieve resolution well in excess of scveral thousands using a variety of ionization sources, including laser ionization and electron impact. An increasingly important property of TOF devices 
has become the ability to measure high mass with high transmission, especially in matrix-assisted laser desorption ionization (MALDI) experiments where masses in excess of $200000 \mathrm{u}$ have been detected using TOF mass spectrometers with high voltage acceleration [14]. In addition, TOF devices are relatively simple, rugged and inexpensive and require only d.c. power supplies with essentially no current load to bias the acceleration region. There are no moving parts, scanning electric fields or slits to limit the throughput.

A major limitation of TOF devices is that there is no storage of ions in the acceleration region. Ion storage is particularly important in studies involving ion/molecule chemistry, for studies of very slow formation of metastables, for enhancing the detection limits by integration of the signal, and for interfacing a continuous ion beam to a TOF device. Although there have been several attempts to store ions using d.c. fields, the ion storage time via this method is limited [15]. However, a more versatile method for achieving ion storage and tandem MS is the ion trap mass spectrometer.

The quadrupole ion trap in itself is a powerful tool for mass analysis and storage of ions over a wide mass range with excellent sample detection limits [18-20]. The ion trap has been used with numcrous ionization sources, including electron impact [19], chemical ionization [21] and photoionization [22], which can create ions directly inside the trap. Alternatively, techniques which require ion introduction from an external source into the trap such as atmospheric pressure sampling glow discharges and electrospray [23] have been interfaced to ion traps. The trap has been used to analyze ions in excess of $70000 \mathrm{Da}$ and recently has been able to achieve extraordinarily high resolution [24]. In addition, an important feature of the trap is its ability to perform multiple stages of tandem mass spectrometry in combination with collisional or photodissociative fragmentation techniques [24]. A key feature of the ion trap is its ability to obtain high sensitivity through ion storage and integration of the signal over an extended period of time [25].
The ion trap also has several inherent disadvantages. Although the trap can store high mass ions, it is often difficult to scan the radiofrequency to a sufficiently large value of the voltage in order to scan out high mass ions. A technique has been developed known as axial modulation to scan out high mass; however, this usually occurs at the expense of the accuracy of the mass calibration $[24,25]$. In addition, very high mass resolution can be achieved in the trap by scanning the r.f. voltage or the r.f. frequency very slowly [26,27]. However, the rate at which the mass is scanned, i.e. $4 \mathrm{~m} / z$ per second in the latter work, is impractical for many applications. The commercial Finnigan ion trap at this time has a resolution of only 185 at $\mathrm{m} / z 69$ and 1700 at $\mathrm{m} / z 502$ and can only scan to $\mathrm{m} / z 650$ at a scan rate of $5000 \mathrm{u} \mathrm{s}^{-1}$ [25].

The goal of our work has therefore been to interface an ion trap storage device as a front end source for a TOF MS [28]. This device combines the storage capabilities of the ion trap with the relatively high resolution, speed and high mass capabilities of the TOF to potentially produce a hybrid instrument with unique capabilities. This article describes the design of an ion trap-time-of-flight device in which ions are initially produced either inside the trap via resonance enhanced multiphoton ionization (REMPI) or external to the trap using an atmospheric pressure d.c. plasma source, low pressure glow-discharge source and electrospray ionization (ESI). Once ions are produced in the trap, they are stored for a time interval ranging from $10 \mu \mathrm{s}$ to $10 \mathrm{~s}$ and subsequently ejected into a RETOF for mass analysis. The ejection method utilizes a d.c. pulse which is applied to the exit endcap of the ion trap. This d.c. pulse destabilizes the trap and the entire contents of the trap are simultaneously ejected for analysis into the RETOF.

In the present article we demonstrate the design and operation of an IT-RETOF MS. The IT is shown to be an effective means of storing ions prior to mass analysis by the RETOF and consequently the storage properties of the IT provide enhanced resolution and sensitivity for analysis 
by TOF. It is also shown that as the storage time is increased from $10 \mu \mathrm{s}$ to $10 \mathrm{~s}$ the resolution is enhanced significantly with the addition of a buffer gas. A resolution of nearly 2100 at $\mathrm{m} / z 93$ can be obtained using the extended storage time provided by the trap, while at $m / z 1000$ a resolution of nearly 3300 can be obtained using an electrospray source. In addition, the IT-RETOF is shown to be an effective means of interfacing a continuous ion beam source to a TOF, achieving a high duty cycle using a low pulse-out extraction rate. A detection limit in the low femtomole regime can be typically obtained for injection from external ionization sources. Further, it is demonstrated that the trap is an effective means of ejecting low mass background prior to analysis by TOF.

\section{Experimental}

The experiment consisted of two modes of operation, trapping internally produced ions and trapping externally produced ions. The experimental setup of the internal ion production experiment is shown in Fig. 1, while that of the external ionization experiments is depicted in Figs. 2-5. Both configurations consisted of a differentially pumped angular RETOF MS (Model D850) interfaced to a quadrupole ion trap storage device (Model C-1251 manufactured by R.M. Jordan Co., Grass Valley, CA) [28] and a liquid injection sample ionization source.

For the internal ionization experiment, a liquid injection sample source was used to deliver the sample, dissolved in a solvent, through a heated stainless steel tube to the first vacuum chamber. In this chamber, pumped to approximately $150 \mathrm{mT}$ Torr, the sample and solvent are vaporized, most of the solvent is removed and the sample passed through a skimmer cone to the ion trap region. In the ion trap, the sample is ionized via $266 \mathrm{~nm}$ radiation generated by a Quanta Ray DCR-3 Nd:YAG laser which passes directly through the trap. The resulting ions are then stored in the trap until the trapping potential is shut off and subsequently an extraction pulse is applied to the exit endcap of the ion trap. The timing and characteristics of the ionizationtrapping-extraction processes are described in detail below. This extraction pulse triggers the start for the TOF mass analysis. Upon leaving the trap, the ion packet enters the field-free drift region $\approx 1 \mathrm{~m}$ long, at the end of which its velocity is slowed and reversed by the ion reflector. The newly focused ion packet then retraverses the drift region and is detected by a dual $40 \mathrm{~mm}$ microchannelplate detector with a gain of about $10^{6}$ to $10^{7}$.

\section{Internal ionization sample injection system}

An ISCO Model $\mu$ LC-500 micropump LC syringe pump was used to deliver the sample through a $50 \mu \mathrm{m}$ fused silica capillary directly to the vaporization chamber. The flow rates were typically $50 \mu \mathrm{L} \min ^{-1}$ and the solvent used was methanol degassed in an ultrasonic bath. The $50 \mu \mathrm{m}$ fused silica capillary was inserted through a zero-deadvolume-tee into a 1 in long, $\frac{1}{16}$ in diameter stainless steel (SS) tube with 0.02 in i.d. A tightly fitting $\frac{1}{8}$ in copper tube was placed over this SS tube and was tightly wound with $\frac{1}{16}$ in thermocoax cable heater. This copper tube was added to the sample introduction assembly to reduce the stress on the wound thermocoax cable heater. The working temperature of the nebulizer was controlled by a thermocouple and a temperature controller. Working temperatures were generally $90-110^{\circ} \mathrm{C}$; however, the actual sample temperature is expected to be much lower due to the expansion and heat carried away by the solvent vapor. This assembly is directly inserted and o-ring sealed to the back of the vaporization chamber (Fig. 1). The vaporization chamber is pumped to a pressure of approximately $150 \mathrm{mtorr}$ by a $650 \mathrm{Lmin}^{-1}$ mechanical pump. The sample vapor is injected from the vaporization region through a skimmer fitted with a 3 in long stainless steel tube ( 0.04 in i.d.) into the ion trap region via the pressure difference. 

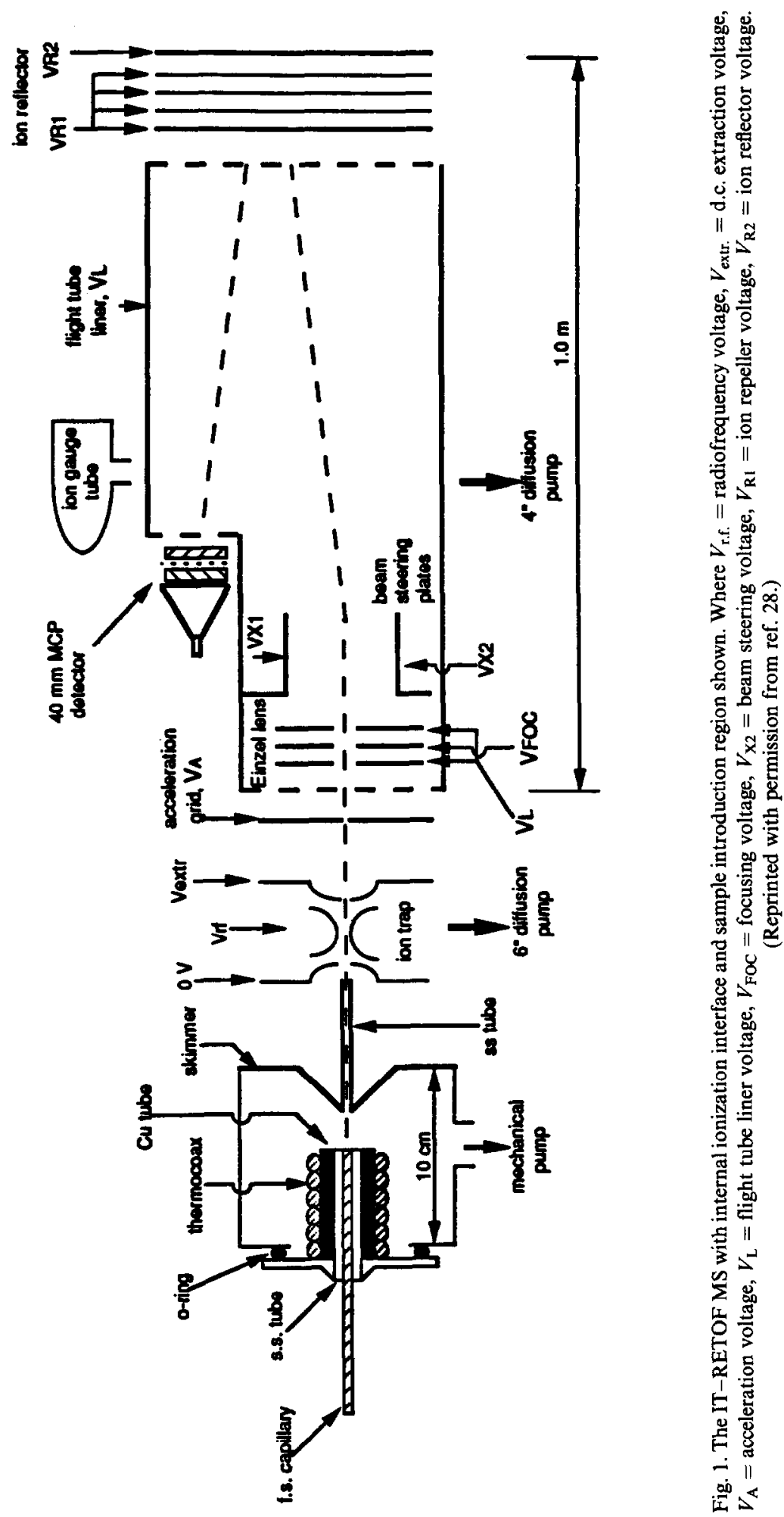


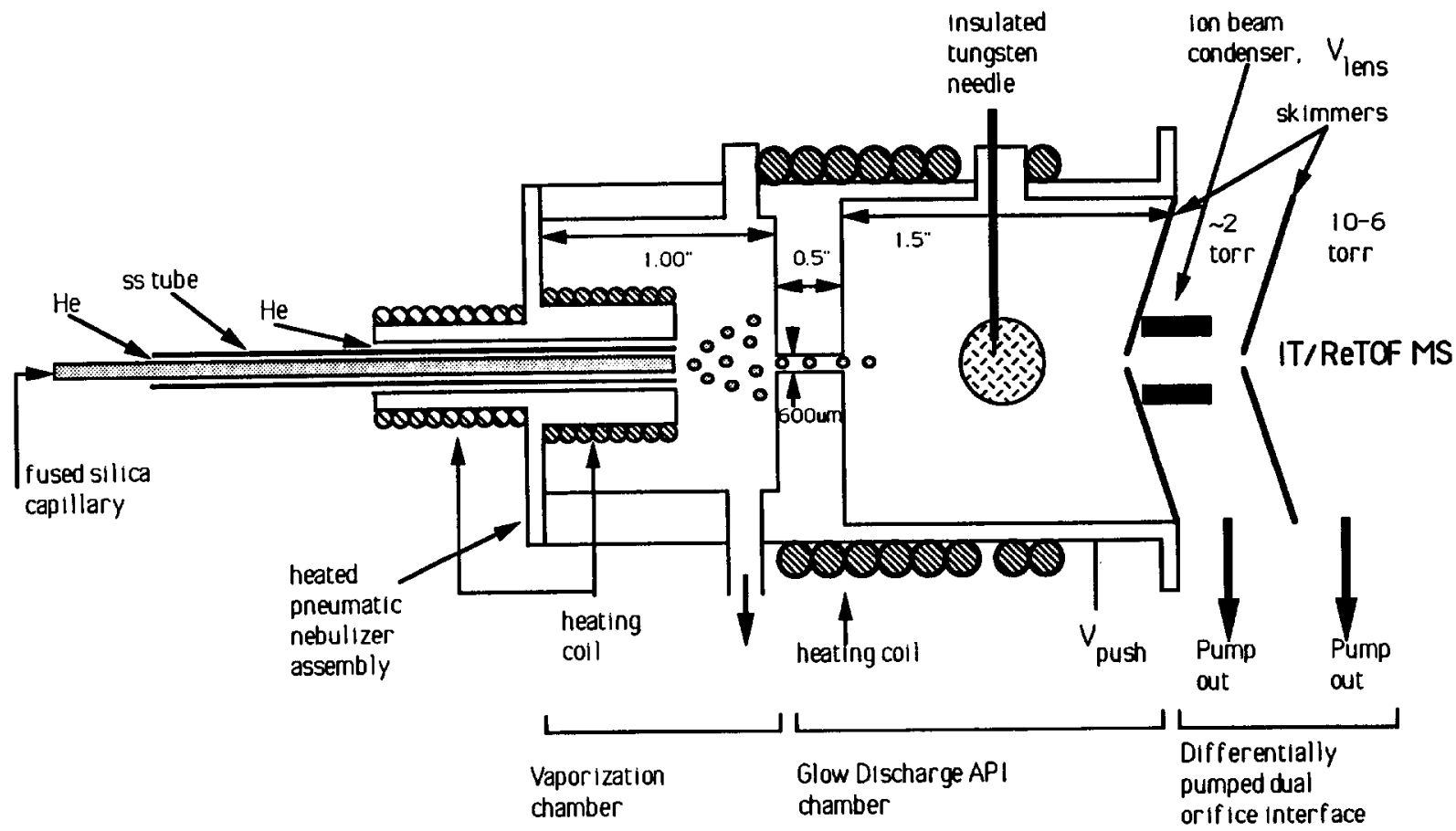

Fig. 2. The sample introduction source including nebulizer, vaporization chamber, API glow discharge chamber, ion beam condenser lens, and differentially pumped dual orifice interface. (Reprinted with permission from ref. 34.)

\section{Internal ionization and trapping}

The sample vapor present in the ion trap is ionized by UV laser radiation $(\lambda=266 \mathrm{~nm})$ which is directed through $3.1 \mathrm{~mm}$ holes in the ring electrode of the ion trap. Ions which are selectively produced by laser-induced REMPI are trapped by the applied r.f. potential, while other ions and unionized sample and solvent vapor are pumped away by the 6 in diffusion pump located directly below the ion trap.

The ion trap consists of two endcap electrodes with a ring electrode between them. These electrodes have hyperbolic surfaces and are configured as shown in Fig. 6. The ion trap was completely enclosed with ceramic spacers placed between the ring and endcaps except for an inlet and exit aperture $3.1 \mathrm{~mm}$ in diameter on the endcaps and two $(3.1 \mathrm{~mm})$ holes for the laser beam to pass through. A $\frac{1}{16}$ in stainless-steel tubing with 0.02 in i.d. was tightly fitted into a hole on the ring electrode in order to introduce helium or other gases into the trap to increase the local pressure when needed. A Vernier needle valve was used to finely control the amount of gas admitted into the trap. Typical pressure in the ion trap ranged from $5 \times 10^{-4}$ to $10^{-3}$ Torr. During operation, both endcaps are held at $0 \mathrm{~V}$ while an r.f. signal of constant frequency $(1.0 \mathrm{MHz})$ and variable amplitude $\left(0-460 \mathrm{~V}_{\mathrm{pp}}\right)$ is applied to the ring electrode. This applied r.f. field serves to trap ions present within the volume of the trap. Varying the r.f. amplitude varies the $m / z$ range of ions that are stable within the trap. Ions with appropriate $m / z$ for a particular r.f. amplitude have a stable trajectory within the trap and, therefore, are trapped [25]. The mass range of the ions that will be trapped was approximated by computer simulation [29]. After a selected $m / z$ range has been trapped, a d.c. pulse was applied to the exit endcap to simultaneously extract all ions from the trap for TOF analysis as detailed below. 

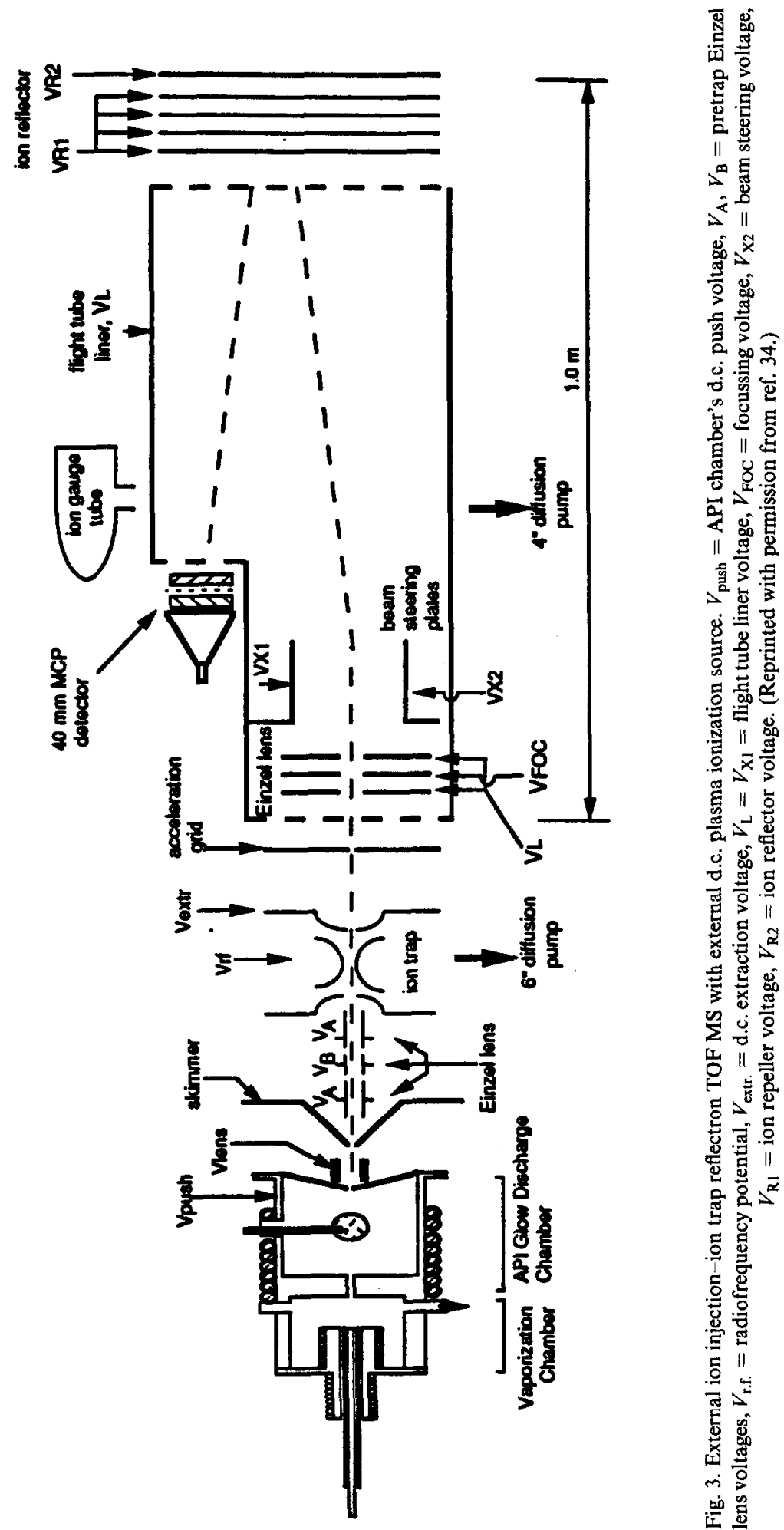


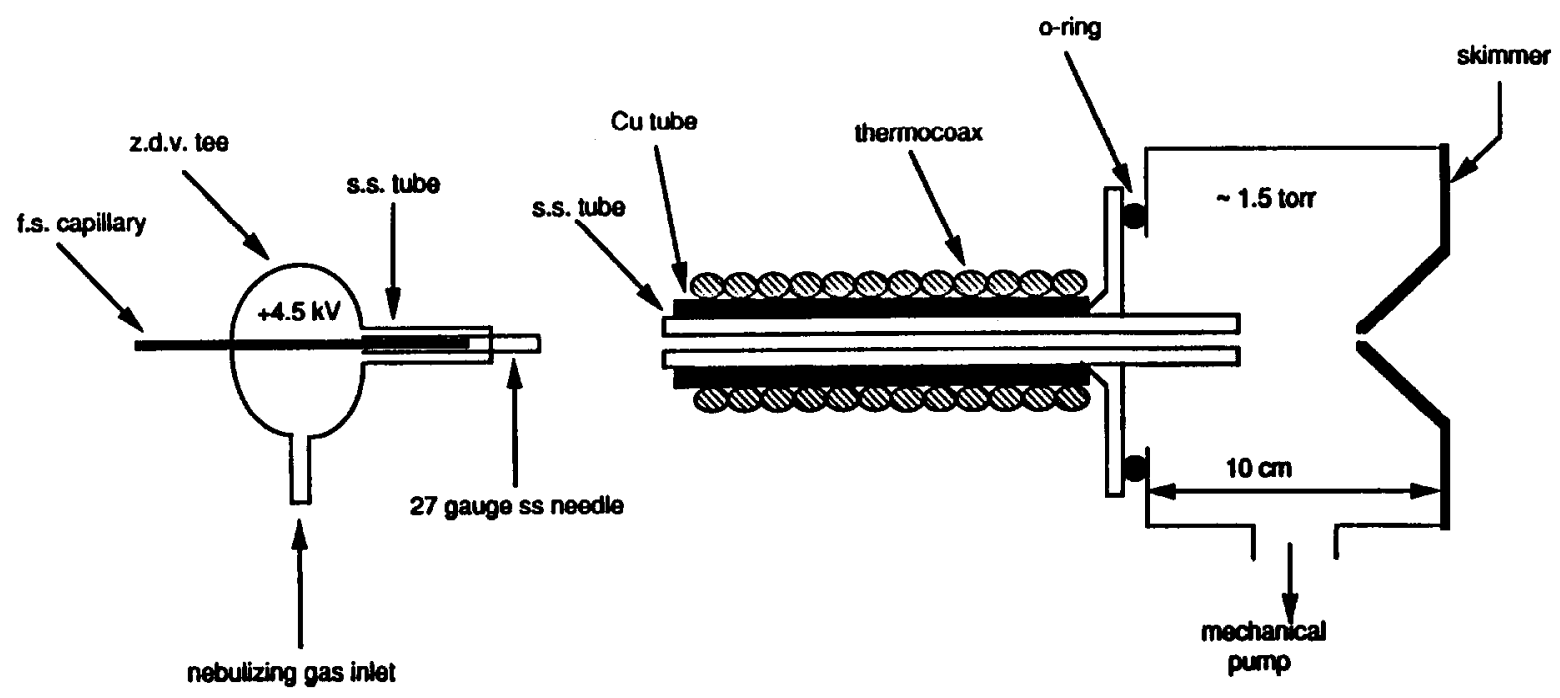

Fig. 4. Detail of the ESI source.

The ionization, trapping, extraction, and detection processes were timed as follows: the Q-switch output pulse of the laser was used to trigger a Systron-Donner Corp. Datapulse 100A Pulse Generator (PG) and a California Avionics Laboratories, Inc. Model 112AR Digital Delay Generator (DDG). The PG output pulse was used to trigger the r.f. power supply (which provided the trapping potential to the ion trap). The DDG output pulse triggered the extraction pulse supply and the LeCroy Model 9400A digital oscilloscope (DOSC) data collection system. In Fig. 7, a typical experimental cycle is depicted and is described in detail with the timing of the internal ionization experiments.

The external ionization and trapping experiments consisted of both a plasma ionization source and an ESI source. For plasma ionization, the liquid injection source was used to deliver the sample, dissolved in a solvent, through a heated pneumatic nebulizer assembly to the vaporization chamber where the sample is vaporized and the solvent removed. The sample then passes through a channel to a separate second chamber and is ionized via a d.c. plasma source in $1 \mathrm{~atm} \mathrm{He}$. The resulting ions are injected through a pair of differentially pumped skimmers $(\approx 1.5$ Torr $)$, which sample the on-axis component of the ion beam. Alternatively, the vaporized sample can be sampled through the first skimmer and ionized in the differentially pumped region via a glow discharge mechanism under reduced pressure. A third method of external ionization used was electrospray which has its own detailed description below. The ions produced externally, regardless of method, were transported into the mass spectrometer region and collimated by a set of Einzel lens into the ion trap device. The ions were stored or accumulated until the trapping potential is shut off and subsequently an extraction pulse is applied to the exit endcap of the ion trap, starting the TOF mass analysis as described above. The timing and characteristics of the trapping-extraction processes are described in detail below.

\section{External ionization sample injection}

In order to inject a variety of samples, including samples of relatively low volatility, into the TOF system, a liquid chromatography-atmospheric pressure ionization interface (LC-API) using a relatively high current plasma source in $1 \mathrm{~atm} \mathrm{He}$ was employed [30]. The system consists of a heated pneumatic nebulizer, a vaporization chamber, a 


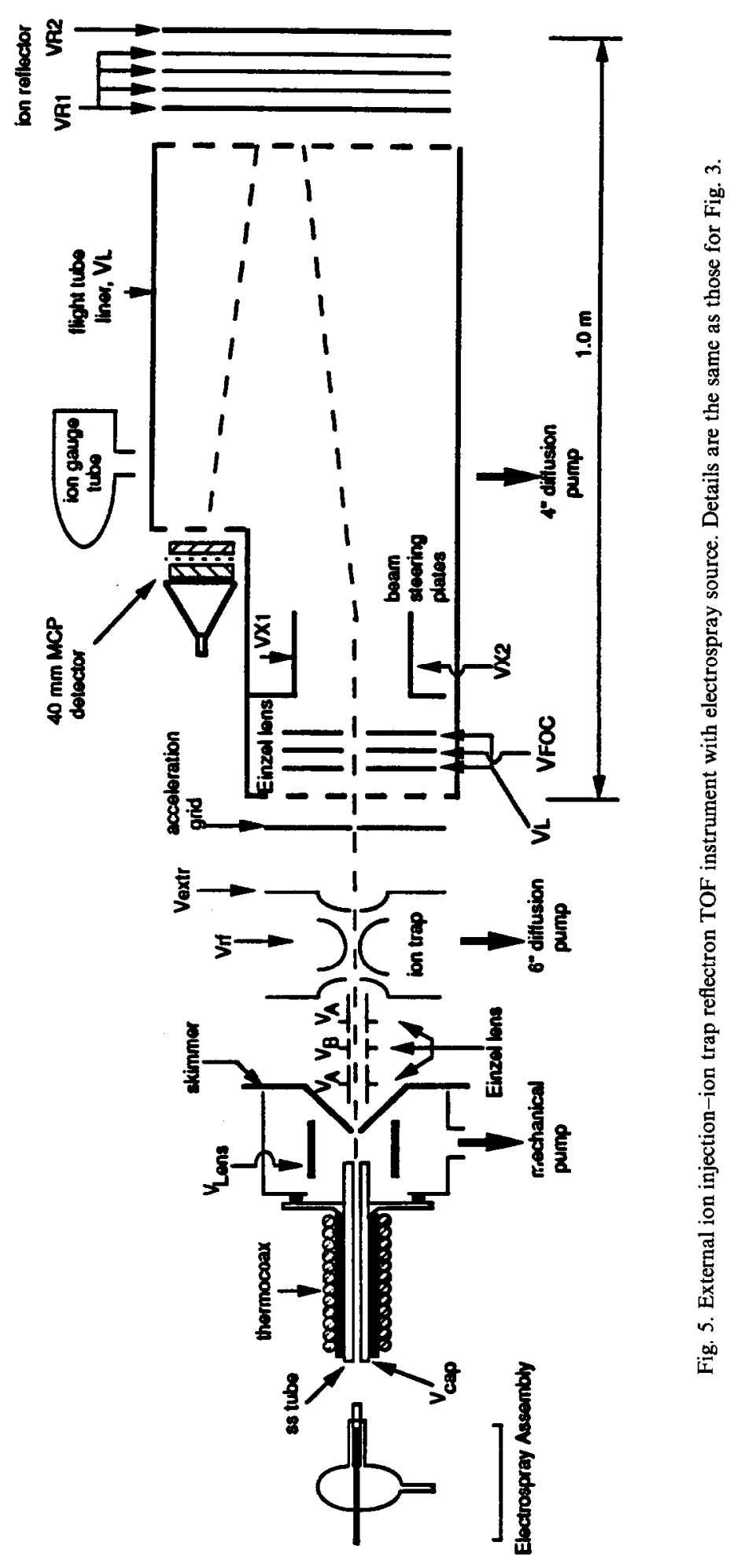




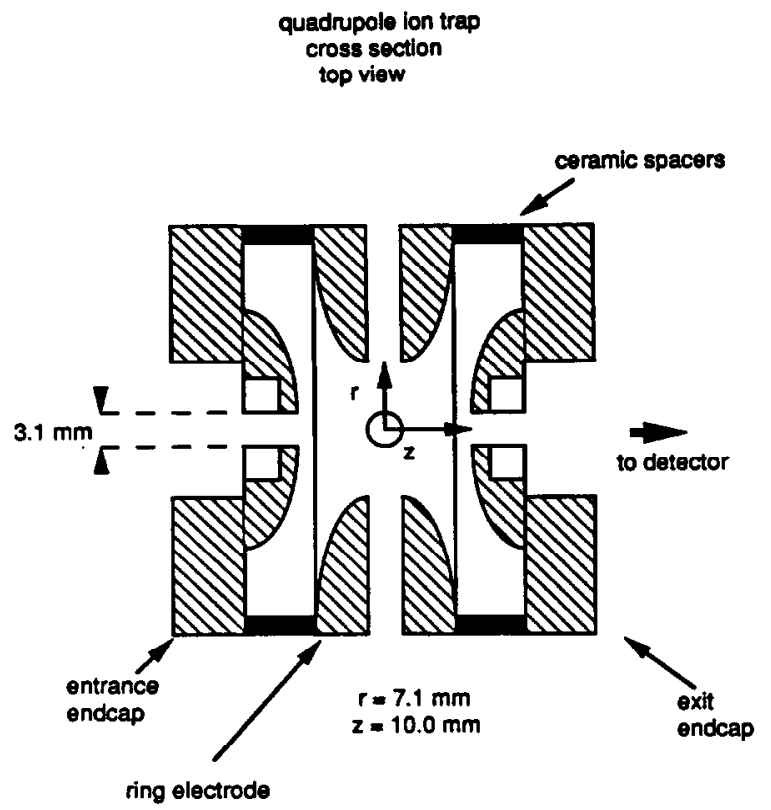

Fig. 6. Detailed construction of the quadrupole ion trap.

plasma source ionization chamber, and a differentially pumped dual orifice interface. The details of the system are as follows (Fig. 3).

An LC syringe pump (Varian Model 8500) was used to deliver the sample through a $180 \mu \mathrm{m}$ fused silica capillary to the vaporization chamber. The flow rates were typically varied from $30 \mu \mathrm{L} \mathrm{min}{ }^{-1}$ up to $0.5 \mathrm{~mL} \mathrm{~min}^{-1}$ and the solvent used was methanol degassed in an ultrasonic bath. The $180 \mu \mathrm{m}$ fused silica capillary was inserted through a zero-dead-volume tee into a coaxial $\frac{1}{16}$ in stainless-steel tube with a 0.01 in i.d. The liquid was pneumatically nebulized into fine droplets using a high velocity jet of helium $\left(1.2 \mathrm{~L} \mathrm{~min}^{-1}\right)$ at the end of the fused silica capillary [30,31]. The generated mist was then swept through a $\frac{1}{8}$ in heated brass tube with another helium gas stream $\left(\approx 200 \mathrm{~mL} \mathrm{~min}^{-1}\right)$ into the vaporization chamber. The helium flow prevents the sample from depositing on the surface of the heated tube and conducts heat to vaporize the solvent. Two fine control flow meters were used to control the gas flow of the nebulizer in order to achieve the best signal. A $\frac{1}{16}$ in thermocoax heater was used heat the $\frac{1}{8}$ in brass tubing. The heated section is a $\frac{3}{4}$ in long, $\frac{1}{2}$ in in diameter cylindrical brass block tightly wound with $\frac{1}{16}$ in thermocoax cable heater through which the $\frac{1}{16}$ in tube is inserted. This assembly is directly oring sealed to the back end of the vaporization chamber. The vaporization chamber consists of a 1.0 in $\times 1.0$ in. diameter cylindrical brass chamber and a heated brass block with a 0.5 in $\times 0.03$ in diameter channel connected to the ionization chamber. The vaporized samples were transferred through the heated narrow channel into the ionization chamber. The temperatures of the pneumatic nebulizer and vaporization chamber can be controlled independently using a thermocouple and temperature controller. The typical working temperature was $150-250^{\circ} \mathrm{C}$. However, the actual working temperature of the sample is expected to be much lower than the body temperature of the vaporization chamber or pneumatic nebulizer since heat is carried away by the vaporized solvent and carrier gas. Because of the solvent vapor and high He flow used in the nebulizer, the actual pressure in this chamber is slightly greater than $1 \mathrm{~atm}$.

\section{External ionization}

Ionization was produced at atmospheric pressure or at reduced pressure in the differentially pumped interface with the plasma ionization source or at atmospheric pressure with the ESI source. The atmospheric pressure ionization process provides soft ionization, where the protonated molecular ion generally is the predominant peak observed. In the reduced pressure ionization process relatively soft ionization or fragmentation can be controlled by altering the experimental conditions.

$A P I$. The ionization chamber (Figs. 2 and 3 ) is directly attached to the skimmer and consists of 1.0 in $\times 0.8$ in (diameter) brass cell with two $\frac{1}{4}$ in Cajon ports with quartz windows for observation and one $\frac{1}{8}$ in Cajon port for the discharge electrode. The glow discharge is formed with the use of a 0.04 in diameter tungsten rod which is ground to a sharp tip. The rod is covered with glass or Teflon 

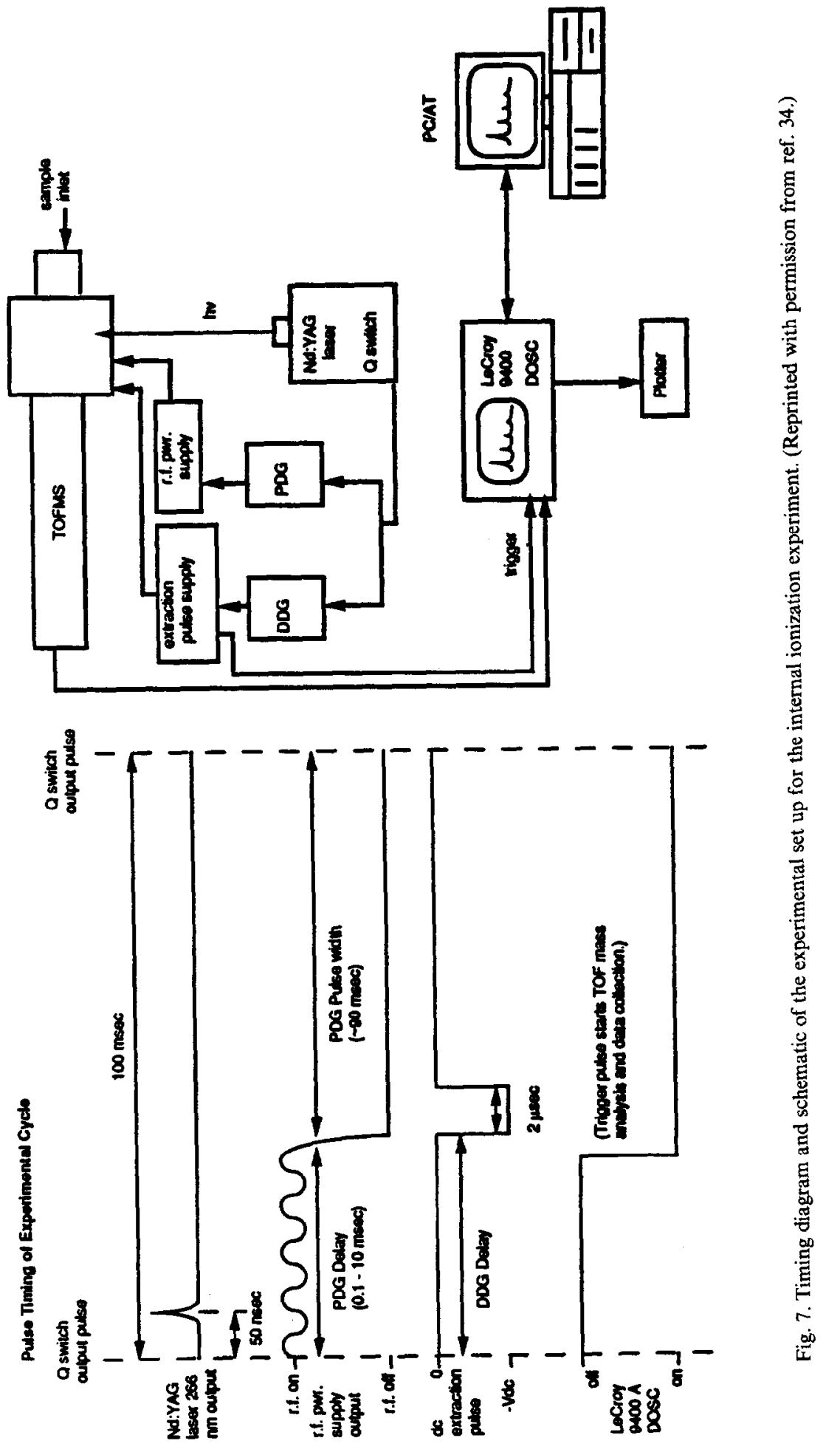
insulation and only the tip is exposed to prevent an unstable discharge from occurring on the side of the rod. In order to initiate the plasma source -1 to $-2 \mathrm{kV}$ was applied to the electrode. When a glow was obtained, the actual operating voltage was dropped as much as $100-500 \mathrm{~V}$ from the initial breakdown voltage and a current of $0.1-0.6 \mathrm{~mA}$ was maintained. The resulting plasma appeared as a white and blue glow extending from the tip of the electrode. The most stable glow was maintained in $1 \mathrm{~atm}$ of helium. The vaporized sample is ionized via ion/molecule reactions induced by the He d.c. plasma source as described in previous work [32]. The ionized sample is transported into a dual-orifice differentially pumped interface. The first sampling orifice of this atmospheric pressure interface is $275 \mu \mathrm{m}$ and the second orifice inlet to the mass spectrometer is $325 \mu \mathrm{m}$. The region between the two orifices is pumped by a $65 \mathrm{~L} \mathrm{~min}^{-1}$ mechanical pump to a pressure of $\approx 1.5$ Torr. The pressure in the mass spectrometer acceleration region under these conditions is $\approx 1 \times 10^{-6}$ Torr. An ion beam condenser with voltage $V_{\text {lens }}$ is placed between the two orifices to enhance the transmission of ions into the mass spectrometer. $V_{\text {push }}$ is a small positive voltage placed on the entire atmospheric presssure interface so that there is a voltage drop between the two orifices.

\section{Reduced pressure ionization. The vaporized} sample traversed through the ionization chamber and was sampled into the differentially pumped region. Instead of initiating a glow discharge at the tip of the electrode in the ionization chamber, -200 to $-500 \mathrm{~V}$ was applied to the entire API chamber to initiate the glow discharge between the first skimmer and the ion beam condenser (maintained at ground potential). Under these conditions a bright glow was formed with a current of $0.1-1.0 \mathrm{~mA}$ and an operating voltage about 100 to $300 \mathrm{~V}$ lower than the initial breakdown voltage. When operated under ambient air conditions, some fragmentation was normally observed in the mass spectrum since higher voltage was required to induce breakdown and maintain the glow. However, if helium was introduced into the system, voltage as low as $-60 \mathrm{~V}$ is sufficient to maintain a glow and thus soft ionization can be achieved. By increasing the discharge voltage, fragmentation can be induced for structural information.

ESI. Another API external ion trapping mode of operation was ESI. In this experiment the ISCO LC syringe pump was used to deliver the sample through a $100 \mu \mathrm{m}$ fused silica capillary directly inserted into the 27 gauge SS needle. This needle was supported in a SS zero-dead-volume tee through which a nebulizing gas flow could be applied. The tee-needle sprayer assembly was maintained at $3.5-4.5 \mathrm{kV}$ relative to the inlet capillary tube. The flow rates were typically $0.5-$ $10 \mu \mathrm{Lmin}^{-1}$ and the solvent used was a mixture of methanol and water of various proportions (50/50-100/0 $\mathrm{MeOH} / \mathrm{H}_{2} \mathrm{O}$ ), determined by solubility requirements of any particular sample. The solvent was degassed in an ultrasonic bath prior to use. All samples were made acidic by addition of acetic acid, as is standard to enhance ionization in positive ion mode electrospray (Figs. 4 and 5).

The sprayer assembly was directed onto a $\frac{1}{16}$ in $200 \mathrm{~mm}$ long $0.5 \mathrm{~mm}$ i.d. heated capillary inlet tube. A tightly fitting $\frac{1}{8}$ in copper tube was placed over this SS tube and was tightly wound with $\frac{1}{16}$ in thermocoax cable heater. The working temperature of the inlet was controlled by a thermocouple and a temperature controller. Working temperatures were generally $70-180^{\circ} \mathrm{C}$ to assist in desolvating the sampled electrosprayed droplets. This assembly is directly inserted into the first vacuum chamber which was pumped to a pressure of approximately 1.5 Torr by a $650 \mathrm{~L} \mathrm{~min}^{-1}$ mechanical pump. The exit of this heated tube is directed onto the previously mentioned $325 \mu \mathrm{m}$ skimmer, i.e. the second skimmer of the set-up in Fig. 3. The inlet tube and skimmer typically had potentials of $50-350 \mathrm{~V}$ and $0-120 \mathrm{~V}$ applied to them, respectively. The distance between the tube and skimmer is adjustable and is usually maintained at $2-7 \mathrm{~mm}$. There is a cylindrical lens 
around the space between the tube exit and skimmer which assists in focussing the ions into the skimmer. This lens has an applied potential of $70-200 \mathrm{~V}$.

The analyte solution is electrosprayed producing highly charged droplets which are pushed toward the inlet tube by electrostatic forces. Some of these droplets enter the tube and pass through it with the help of the tube's applied potential and the ambient pressure gradient. The ions exiting the tube are then drawn into the skimmer via the potential difference between the tube and the skimmer. In this space the ions undergo many collisions and by altering the potential difference, the pressure and the gap length, fragmentation can be enhanced or reduced [33].

\section{Trapping}

The ions produced by the external ionization sources were sampled through the second skimmer $(325 \mu \mathrm{m})$ and entered the mass spectrometer region. The ions underwent a supersonic jet expansion and cooling, then were collimated by a set of Einzel lens into the ion trap device.

The ion trap is the same as in the internal ionization experiment except the two holes that allowed the laser beam to pass through the trap are closed with Teflon plugs. In some cases, if the energy of the ions from the ionization source is high, the ring electrode and both endcaps can be biased with positive voltage to slow down the ions and improve the trapping efficiency. Using this method, ions with energy as high as $300 \mathrm{eV}$ can be injected into and stored in the trap as will be discussed in more detail later.

The trapping, extraction, and detection processes were timed as follows. A Global Specialties Co., 4001 pulse generator (PG) was used to trigger two California Avionics Laboratories, Inc. model 112 AR digital delay generalors (DDG1 and DDG2). The DDG1 was used to trigger the r.f. power supply. An R.M. Jordan Co. r.f. power supply operated at $1.0 \mathrm{MHz}, 0-460 \mathrm{~V}_{\mathrm{pp}}$ was used to trap ions up to $m / z 185$, while a modified EAI r.f. power supply with variable amplitude $0-2200 \mathrm{~V}_{\mathrm{pp}}$, 1.1 $\mathrm{MHz}$ output was used to trap ions with $\mathrm{m} / \mathrm{z}$ greater than 200. The DDG2 output pulse triggered the extraction pulser and this pulser passed its pulse to trigger the Lecroy model 9400A DOSC or Precision Instrument Inc. model 9825 Signal Averager (SA) (see Fig. 8). The signal from the detector was amplified by a Stanford Research Systems Model SR445 DC-300 MHz amplifier when needed.

In Fig. 8, a typical experiment cycle for external ionization is depicted: the PG $(+10 \mathrm{~V})$ triggered both DDGs. The r.f. trapping potential remains on while this sequence occurs, thus trapping the ions continuously produced from the external ionization source using the buffer gas to collisionally relax the energetic ions produced by this source. The r.f. potential remains on until the DDG1 pulse triggers the r.f. power supply. Then, $2 \mu \mathrm{s}$ (internal delay of r.f. power supply) after the rising edge of the DDG1 pulse arrives, the r.f. potential is shut off and remains off for the entire pulse width of the DDG1 pulse. DDG1 output pulses (20 ns rise time, $+10 \mathrm{~V}$ amplitude, $10 \mu \mathrm{s}$ FWHM) can be delayed from a few microseconds to as long as $10 \mathrm{~s}$ after triggering. Therefore, the delay of the DDG1 determined the duration of ion trapping, i.e., how long the ions could be stored or accumulated in the trap. After its set delay, the DDG2 outputs a pulse ( $20 \mathrm{~ns}$ rise time, $+10 \mathrm{~V}$ amplitude, $10 \mu$ sWHM) which triggers the extraction pulser. The extraction pulser serves the dual purpose of both providing a start time reference for the TOF mass analysis and providing an extraction pulse to the exit endcap of the ion trap, thereby ejecting the ions from the trap. Upon the arrival of the rising edge of the DDG2 pulse, the extraction pulser passes this trigger pulse, the DDG2 output pulse, to the DOSC or SAs external trigger. This provides the start time reference for the TOF mass analysis. Following an interval of $1.5 \mu \mathrm{s}$ after the arrival of the rising edge of the DDG2 output pulse, the extraction pulser sends the extraction pulse to the exit endcap of the ion trap. This extraction pulse was a d.c. square wave $-150 \mathrm{~V}$ in amplitude and $2 \mu \mathrm{s}$ in width 


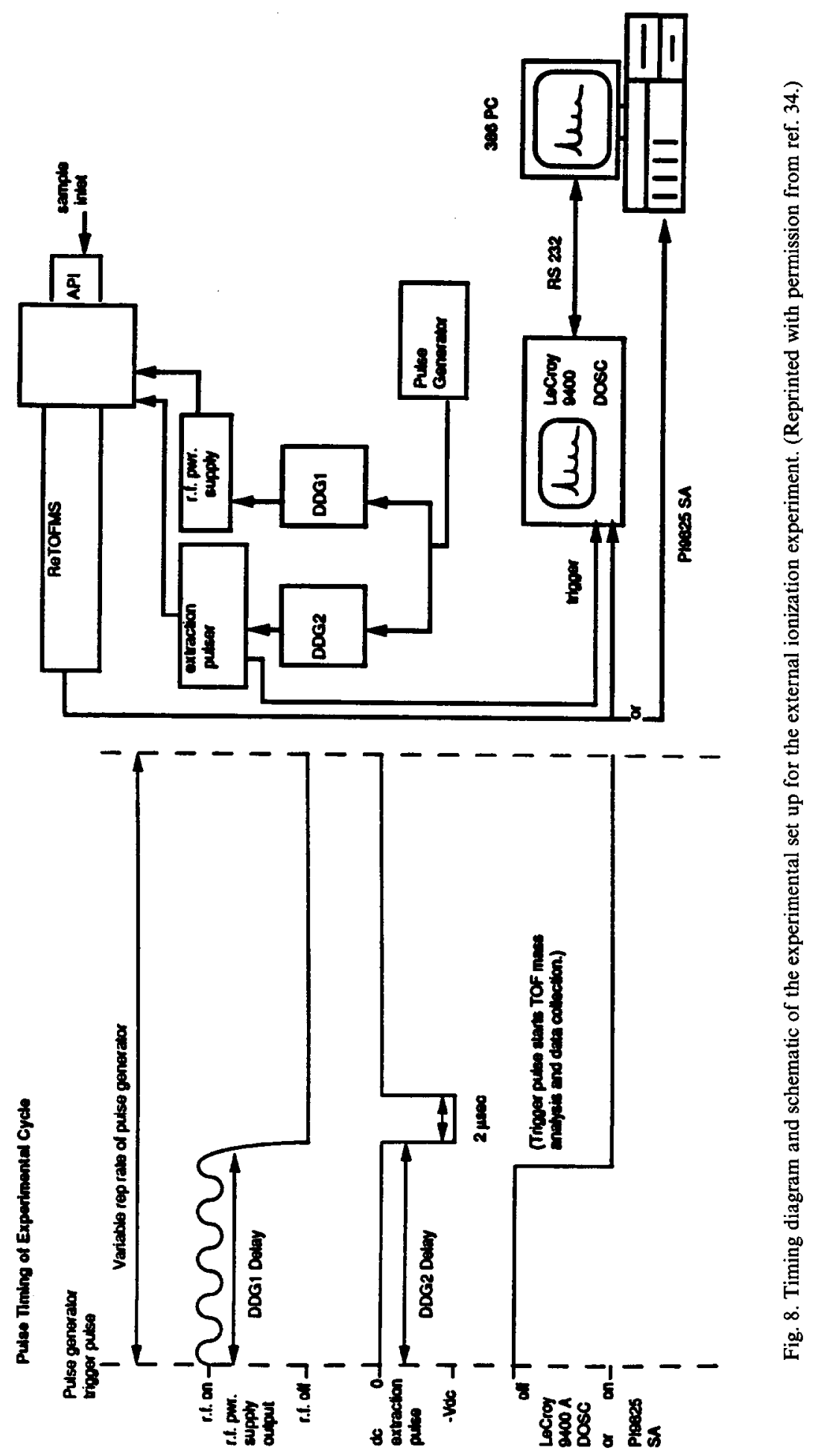


with $10 \mathrm{~ns}$ rise and fall times. The delay of DDG2 was set to coincide with the delay of DDG1, therefore, extraction of the ions occurred simultaneously with the r.f. trapping potential being triggered off. The repetition rate of the pulse generator can be set at $0.1-10 \mathrm{KHz}$, limited by the ions with the longest flight time, therefore the repetition rate of the entire experimental cycle varies from 0.1 to $10 \mathrm{KHz}$.

In the internal ionization experiment the timing details were the same with the following exceptions (see Fig. 1). The Q-switch output pulse from the Nd: YAG laser triggered the PG and the DDG. After a $50 \mathrm{~ns}$ internal delay the laser pulse fires ( $7 \mathrm{~ns}$ FWHM at $266 \mathrm{~nm}$ ) and ionizes the sample vapor in the ion trap. The r.f. trapping potential remains on while this sequence occurs, thus trapping the ions produced by the laser beam. The PG pulse triggers the r.f. power supply so that the delay of the PG determined the duration of ion trapping. The DDG triggered the extraction pulse supply which serves the dual purpose of both providing a start time reference for the TOF mass analysis and providing an extraction pulse to the exit endcap of the ion trap, thereby ejecting the ions from the trap.

\section{TOF Operation}

The ions upon exiting the ion trap, pass through a set of accelerating plates and an Einzel lens which serve to focus the ion packet and accelerate it into the field-free flight tube region through a potential difference of about $-1400 \mathrm{~V}$. A pair of beam deflecting plates are then used to steer the ions towards the ion repeller-reflector assembly, where the ion packet is more tightly focused, reversed in direction, and reaccelerated through the flight tube (with angular displacement from its initial axis of trajectory) onto the $40 \mathrm{~mm}$ dual microchannel plate detector (Model C726, R.M. Jordan Co.). In more recent work, the detector has been updated to a $40 \mathrm{~mm}$ triple microchannelplate detector (Model C726-T, R.M. Jordan Co.) for enhanced sensitivity. The reflectron flight tube is pumped by a
Varian VHS 4 diffusion pump while the main chamber is pumped by a Varian VHS 6 diffusion pump. A restriction of 1 in tubing is placed between the flight tube and the main chamber, which produced typical operating pressures of $8 \times 10^{-6}$ and $1 \times 10^{-6}$ Torr, respectively. The actual pressure in the ion trap during sample introduction though was between $5 \times 10^{-4}$ and $10^{-3}$ Torr.

The TOF of the extracted ion packets was measured on the DOSC. Signal averaging was used to enhance the signal-to-noise ratio and reported spectra are averages of 100 single waveforms unless noted otherwise. In the experiment, DDG2 simultaneously triggered the DOSC and the extraction pulser. The ion signals from the detector were sent to the input of the DOSC, and the time difference between various ion peaks and the trigger $(t=0)$ reference provides the time of flight of each ion.

The TOF spectra in the DOSC are then transferred to a 386 IBM compatible PC using an RS 232 interface bus established between the DOSC and the computer. A user written QUiCKBAsIC program was used to control the transfer processes. The size for each data point was 16 bit for our experiments. The raw data from DOSC were in ASCII form. It was converted into signed decimal form by a user-written program.

A study of system resolution performance found that the spectral resolution is limited by the time resolution of the Lecroy 9400A DOSC (10 ns). Later experiments used a Precision Instrument Inc. PI9825 SA in order to take advantage of its $5 \mathrm{~ns}$ time resolution. The PI9825 model is a complete SA on two PC-AT cards inserted onto a 386/ 486 PC motherboard with a maximum digitization rate of 200 Msample per second. Software accompanying this product provides a graphic control panel used to acquire and store the data on a real-time basis. The stored data in binary form was converted into ASCII form by a user-written program and loaded into commercial spreadsheet software such as EXCEL or LOTUs for further data analysis.

Mass calibration was performed by measuring 


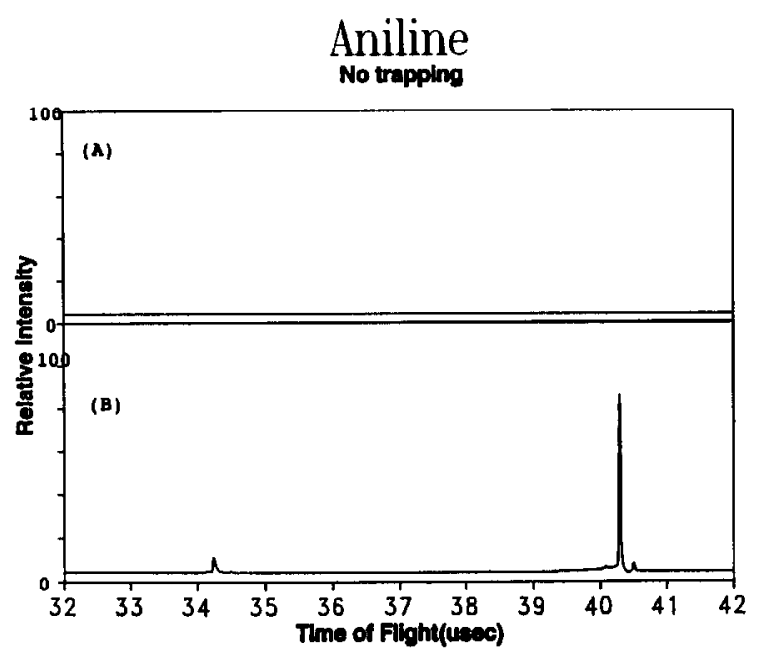

Fig. 9. IT-RETOF mass spectra of aniline with no r.f. trapping potential applied. (A) $20.10 \mu$ s delay before extraction; (B) no delay before extraction. Both spectra were obtained with the following operating conditions. $V_{\mathrm{L}}=V_{\mathrm{X} 1}=-1100 \mathrm{~V}$, $V_{\mathrm{X} 2}=-980 \mathrm{~V}, \quad V_{\mathrm{FOC}}=-400 \mathrm{~V}, \quad V_{\mathrm{A}}=-300 \mathrm{~V}, \quad V_{\text {extr. }}=$ $-150 \mathrm{~V}, V_{\mathrm{R} 1}=+1200 \mathrm{~V}, V_{\mathrm{R} 2}=+1950 \mathrm{~V}$. (Reprinted with permission from ref. 28.]

the time of flight $(T)$ of a few known masses (such as background water or methanol cluster ion signal) to find out the constant $x$ and $y$ in the empirical equation by linear regression analysis, $m / z=x T^{2}+y$.

\section{Results and discussion}

\section{Internal ionization}

Mass spectra. The capabilities of the ion traptime-of-flight device are demonstrated in the mass specra of Figs. 9-12. These spectra were taken with $7 \times 10^{-4} \mathrm{M}$ aniline dissolved in methanol. Sample and solvent were pumped into the interface chamber via the syringe pump set at a flow rate of $50 \mu \mathrm{Lmin}^{-1}$. Vaporization, delivery to the ion trap region, and ionization occurred as described above. Figures 9(A) and 9(B) are mass spectra of aniline without ion trapping, i.e. no r.f. applied to the ring electrode of the ion trap. Figure 9(B) shows the extraction and TOF mass analysis of aniline ions by applying a $-150 \mathrm{~V}$ d.c. pulse to the exit endcap of the ion trap $0.10 \mu$ s after laser ionization

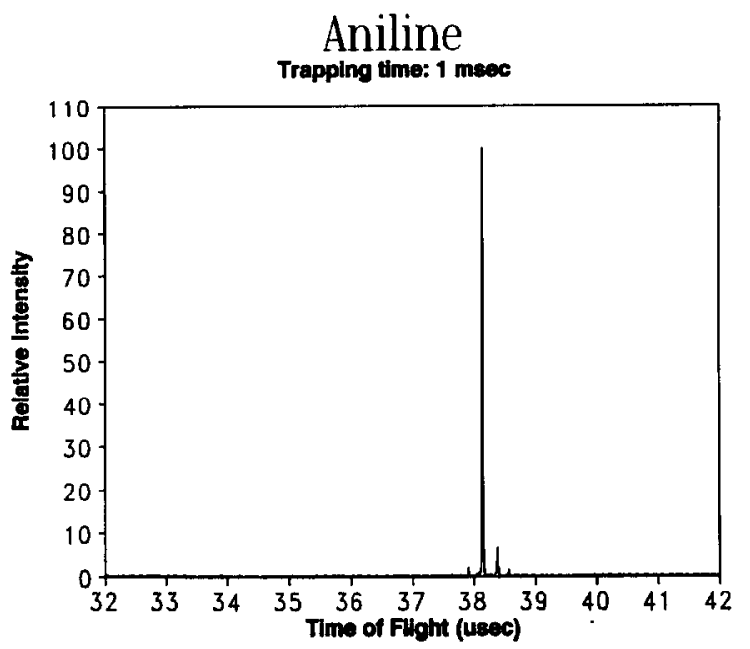

Fig. 10. IT-RETOF mass spectrum of aniline obtained by laser induced REMPI at $266 \mathrm{~nm}$ with $1.0 \mathrm{~ms}$ trapping time. R.f. frequency $=1.19 \mathrm{MHz}$, r.f. voltage $=180 V_{p p}$, and all other operating conditions the same as those of Fig. 9. (Reprinted with permission from ref. 28.)

has occurred. The $m / z 93$ peak is assigned to the aniline molecular ion. The ${ }^{13} \mathrm{C}$ isotope peak is present at $\mathrm{m} / z 94$ at approximately $6 \%$ of the molecular ion peak's intensity. The $m / z 77$ peak is the $\mathrm{C}_{6} \mathrm{H}_{5}^{+}$fragment. Increasing the laser power increases the relative intensity of the $\mathrm{C}_{6} \mathrm{H}_{5}^{+}$peak. In Fig. 9(A) the spectrum is of the same sample and conditions as Fig. 9(B) except that the extraction pulse is delayed $20.10 \mu \mathrm{s}$. This spectrum shows that with no r.f. applied to the ring electrode of the ion trap, all ions produced are pumped away by the 6 in diffusion pump, thus, only a flat line background was detected. Figure 10 shows a spectrum of the same sample with the r.f. potential applied to the ring electrode of the ion trap and an extraction delay of $1 \mathrm{~ms}$ ( $1 \mathrm{~ms}$ trapping time). This spectrum clearly illustrates that the ions are successfully trapped and the trapping results in an increase in resolution.

The ability of the IT-RETOF mass spectrometer to trap ions for an extended period of time is shown in Fig. 11. In Fig. 11 an aniline spectrum with $2 \mathrm{~s}$ trapping time using a laser repetition rate of $0.5 \mathrm{~Hz}$ results in a similar mass spectrum with similar resolution as that with a $1 \mathrm{~ms}$ trapping time. 


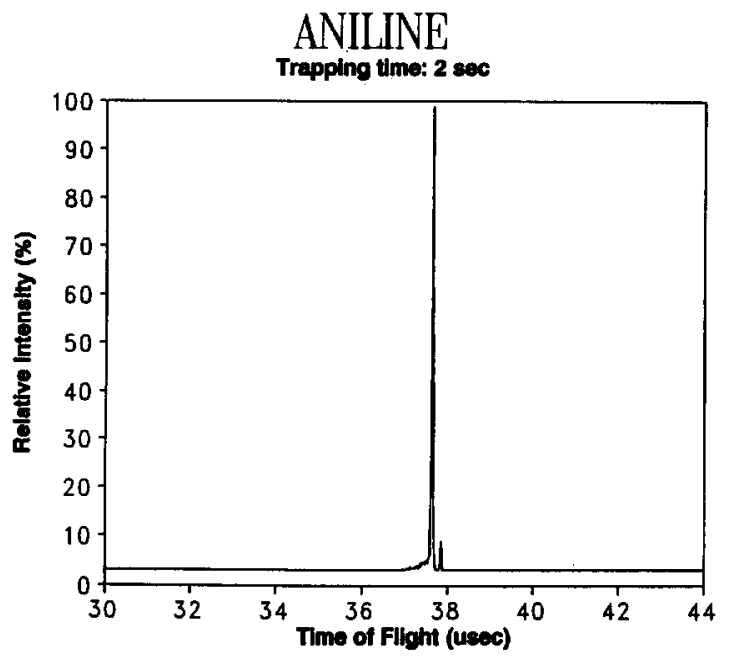

Fig. 11. IT-RETOF mass spectrum of aniline obtained by laser induced REMPI at $266 \mathrm{~nm}$ with $2 \mathrm{~s}$ trapping time. All operating conditions were the same as those in Fig. 7 except the laser repetition rate was reduced to $0.5 \mathrm{~Hz}$. (Reprinted with permission from ref. 28.)

Although the mass spectra in Figs. 10 and 11 are similar, the relative peak height after $2 \mathrm{~s}$ of storage typically decreases by a factor of 5 to 10 relative to storage after $1 \mathrm{~ms}$ as ions are lost from the trap. In Fig. 12 is shown a laser REMPI mass spectrum of aniline obtained when the laser power is increased to produce extensive fragmentation. This mass spectrum demonstrates that the molecular ion and its accompanying fragments can all be stored for an extended time, i.e. $5 \mathrm{~ms}$, by the trap and detected by the RETOF. The laser REMPI mass spectrum is essentially the same as that obtained without trapping. The resolution in this figure is slightly degraded compared to the other figures due to the extended mass range processed by the digitizer. An expanded view of any of these peaks reveals a resolution very similar to that obtained in Figs. 9-11.

\section{Experimental parameters}

$R . f$ frequency and voltage. The r.f. potential applied to the ring electrode determined the $\mathrm{m} / \mathrm{z}$ range that resulted in stable trajectories in the trap, that is, the applied r.f. frequency determined

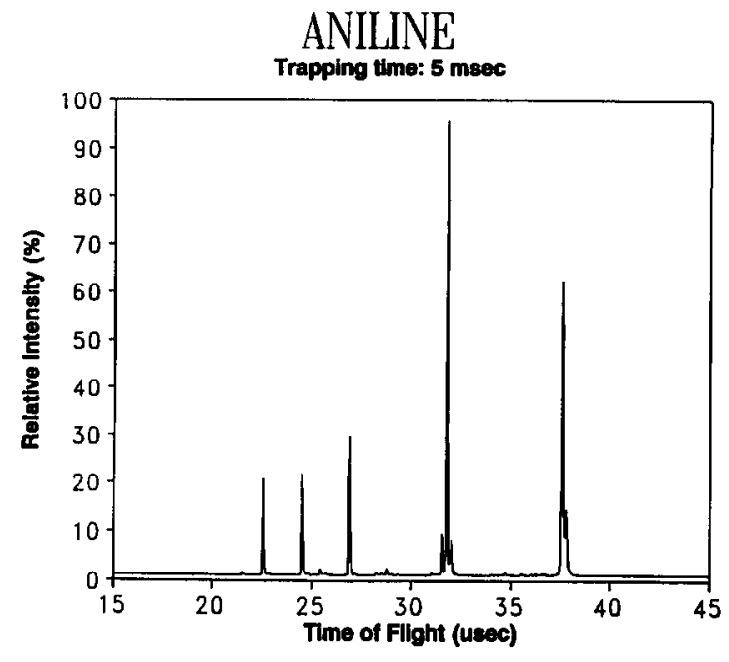

Fig. 12. IT-RETOF mass spectrum of aniline obtained by laser induced REMPI at $266 \mathrm{~nm}$. The laser power has been increased to induce fragmentation which is stored in the trap for $5 \mathrm{~ms}$ and detected by the RETOF MS. All operating conditions were the same as those in Fig. 7. (Reprinted with permission from ref. 28.)

the $m / z$ range trapped. Varying the r.f. frequency was experimentally determined to move the observed $\mathrm{m} / \mathrm{z}$ range that was trapped. As the frequency was lowered, a region of lower $\mathrm{m} / \mathrm{z}$ ions were trapped and as the frequency was increased this "window" shifted toward higher $m / z$. Other than a shift in $m / z$ values over which the "window" was located, frequency variations showed no changes in instrumental performance in the experiment. The experimental results are reported with $1.19 \mathrm{MHz}$ as the selected r.f. frequency, as this setting was compatible with our chosen $m / z$ range (i.e. the "trapping window" roughly centered on $m / z$ 93). Frequencies in the range $800 \mathrm{kHz}-$ $1.19 \mathrm{MHz}$ were studied. Varying the amplitude of the r.f. voltage applied at a fixed frequency also served to move the "trapping window." A limitation observed here was that r.f. voltage amplitudes less than $160 \mathrm{~V}_{\mathrm{pp}}$ were too low to trap the ions studied in this work.

D.c. extraction pulse. The extraction voltage was varied from -50 to $-350 \mathrm{~V}$. It was found that $-150 \mathrm{~V}$ provided both the best signal intensity 
and resolution. If the extraction voltage was increased significantly, both the resolution and signal were degraded. This is due to the fact that the reflectron performance is very sensitive to the exact voltages used on the grids. Various extraction pulse widths were also attempted. A pulse width of approximately $0.5 \mu \mathrm{s}$ or less was too short to allow significant extraction of ions from the trap, resulting in decrcascd signal intensity or no observed signal. A pulse width from $>0.5 \mu$ s to $\approx 5.0 \mu \mathrm{s}$ resulted in the best signal intensities, with $\approx 2.0 \mu$ s yielding the best resolution.

Resolution and sensitivity. The peak width (FWHM) of the molecular ion peak in Fig 9(B), where there was no trapping, was $30 \mathrm{~ns}$. This corresponds to a resolution of nearly 700 . In Fig. 10, the molecular ion peak width was $16 \mathrm{~ns}$, which is a resolution of 1300 at $m / z$ 93, when the trapping capability is utilized. This enhanced resolution provided by the ion trapping is probably due to improved spatial resolution of the ions. As shown in recent computer simulations, the extended trapping of ions results in their accumulation in the center of the trap [29]. Upon d.c. ejection, this accumulation results in a marked improvement in the spatial resolution factor in the TOF device, as opposed to the untrapped situation where the ions are produced over the diameter $(\approx 2 \mathrm{~mm})$ of the laser ionization beam. A well-defined ion packet in a narrow region of space might yet prove to be particularly suited for use in obtaining high resolution in a gridless reflectron device.

A measurement of the sensitivity was also performed in the IT-RETOF using laser induced REMPI at $266 \mathrm{~nm}$ for benzene. The benzene was dissolved in methanol in a concentration of $10^{-5} \mathrm{M}$ and a series of successive dilutions down to $10^{-7} \mathrm{M}$ were made. The sample was injected as before using the microsyringe pump at a rate of $30 \mu \mathrm{L} \mathrm{min}-1$ into the trap. The $266 \mathrm{~nm}$ radiation was used at a power density of $5 \times 10^{5} \mathrm{~W} \mathrm{~cm}^{-2}$ which was the highest power at which ionization could be produced without significant fragmentation. In the case where no storage was used, a lower limit of detection of $\approx 600 \mathrm{fmol}(S / N=3)$ was obtained. If a storage time of $90 \mathrm{~ms}$ was used following a single laser pulse then the detection limit was $\approx 200 \mathrm{fmol}$, i.e. at least a factor of 3 improvement over no trapping. This is probably partially due to the smaller ion cloud following trapping which is more efficiently ejected along the $z$-axis of the trap. However, if the ions are trapped for $1 \mathrm{~s}$ and the laser pulsed ten times during this period, then a detection limit of $\approx 60 \mathrm{fmol}$ is achieved. Thus, the production and storage of ions over several laser pulses enhances the sensitivity by a factor of nearly 10 compared to the situation where no storage is used. Of course, during this storage time some fraction of the ions will be lost from the trap and at some point saturation of the trap with resulting space charge losses will result. Indeed it is found that a saturation point is reached after storage of about 4 pulses. Additional pulses do not appear to increase the detection limit while inducing space charge broadening at the given laser power. However, at lower laser power where less ions are produced per laser pulse or in a sample molecule where ionization is much less efficient than benzene, the use of additional laser pulses within a storage time interval will be increasingly important for enhancing the sensitivity for detection. Nevertheless, in the case illustrated herein, the use of ion trap storage with the RETOF can provide excellent sensitivity and may enhance the sensitivity even further where low signal levels are present.

\section{External injection}

API. The capabilities of the IT-RETOF for detection of ions generated externally by the API d.c. plasma source are demonstrated in Figs 13-15 [34]. Figure 13 shows the API mass spectrum of the molecular ion region of pyridine injected into the IT-RETOF using a $10^{-6} \mathrm{M}$ pyridine solution in methanol. The vaporization and ionization of the liquid sample and the transmission of the resulting ions to the ion trap were performed as described previously. The spectrum obtained reveals three fully resolved peaks at $m / z 78,79$ and 80 which 


\section{Atmospheric Pressure lonization of Pyridine, MW 79, 950 ms trapping time, Resolution $>1500$}

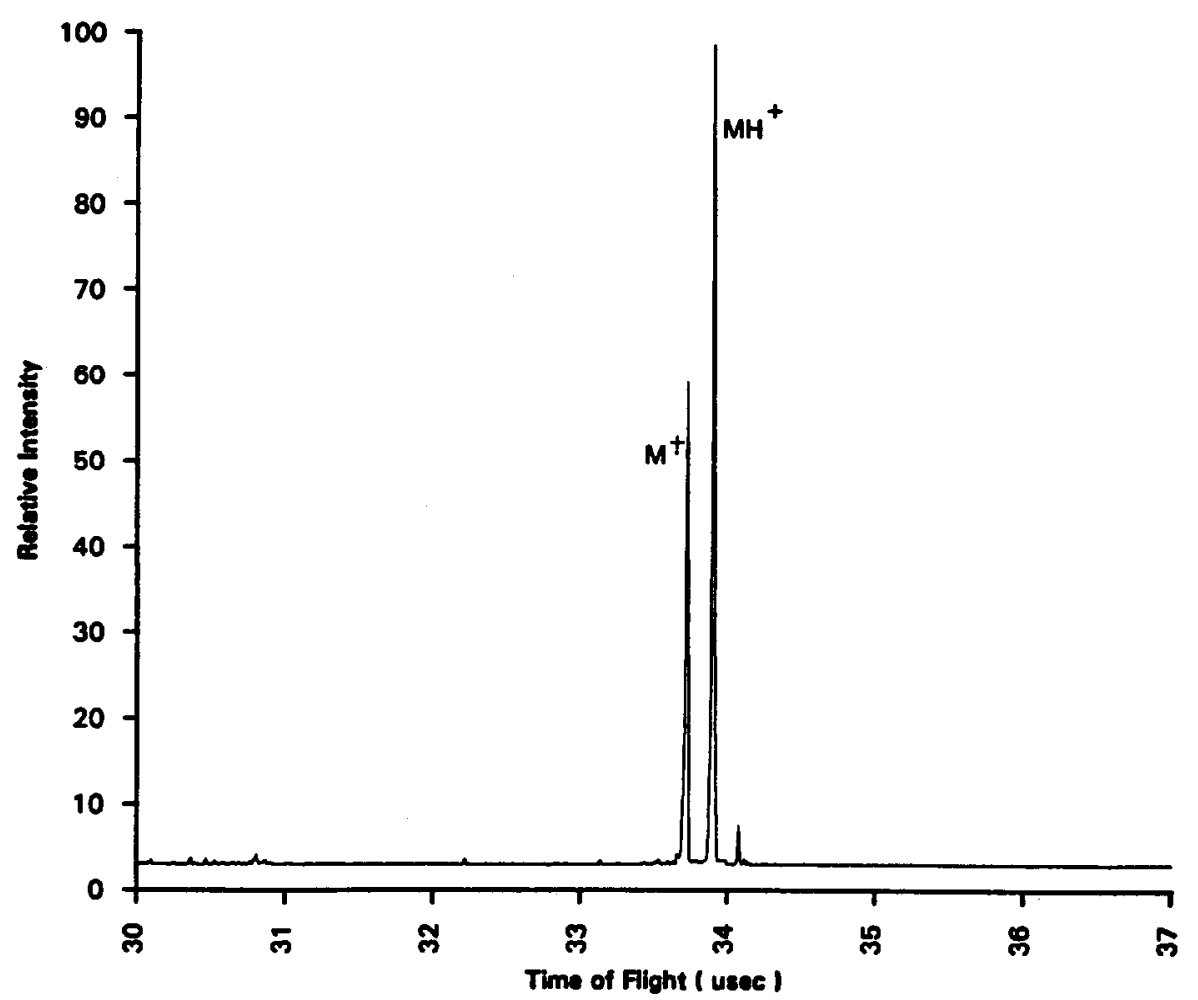

Fig. 13. API mass spectrum of pyridine with $V_{\text {push }}=0 \mathrm{~V}, V_{\text {lens }}=+200 \mathrm{~V}, V_{\mathrm{A}}=+90 \mathrm{~V}, V_{\mathrm{B}}=+190 \mathrm{~V}, V_{\mathrm{rf}}=+400 V_{\mathrm{pp}}, V_{\text {extr. }}=-300 \mathrm{~V}$, $V_{\mathrm{L}}=V_{\mathrm{X} 1}=-1500 \mathrm{~V}, V_{\mathrm{FOC}}=0 \mathrm{~V}, V_{\mathrm{X} 2}=-1385 \mathrm{~V}, V_{\mathrm{R} 1}=+110 \mathrm{~V}, V_{\mathrm{R} 2}=+1000 \mathrm{~V}, V_{\text {bias }}=+280 \mathrm{~V}$. (Reprinted with permission from ref. 34.)

are assigned to the molecular ion, the protonated molecular ion and its ${ }^{13} \mathrm{C}$ isotopic peak respectively. This spectrum was obtained for a storage time of $950 \mathrm{~ms}$ and the resulting resolution was $>1500$. In this case the R.M. Jordan r.f. power supply was used to supply r.f. voltage to the ring electrode. A voltage of $\approx 400 \mathrm{~V}_{\mathrm{pp}}$ was used at a frequency of $1.0 \mathrm{MHz}$ in this spectrum. The r.f. voltage is pulsed off before the ions are ejected into the RETOF which results in a peak width FWHM of $11 \mathrm{~ns}$ and thus relatively high resolution is observed. The sensitivity here is also excellent where only $160 \mathrm{fmol}$ of analyte was used to obtain this signal. The ability to pulse off the r.f. power supply prior to d.c. ejection provides better resolution in this spectrum than if the r.f. is not pulsed off. However, the maximum voltage of 460 $\mathrm{V}_{\mathrm{pp}}$ available for this supply limits the ability to trap externally injected ions [34] from the d.c. plasma source to $m / z 185$.

In Fig. 14 is shown API d.c. plasma source IT-RETOF mass spectra of amitriptyline. The spectrum in Fig. 14 was obtained using $10^{-6} \mathrm{M}$ amitriptyline dissolved in methanol which was vaporized and ionized as described previously. The mass spectrum obtained consists of the protonated molecular ion peak at $m / z 279$ and its ${ }^{13} \mathrm{C}$ isotopic peak at $\mathrm{m} / z$ 280. This spectrum was obtained using a modified EAI r.f. quadrupole power supply operating at $1.1 \mathrm{MHz}$ and $530 \mathrm{~V}_{\mathrm{pp}}$. This unit could supply the additional r.f. voltage, up to $2200 \mathrm{~V}_{\mathrm{pp}}$, required to trap $m / z>185$. This 


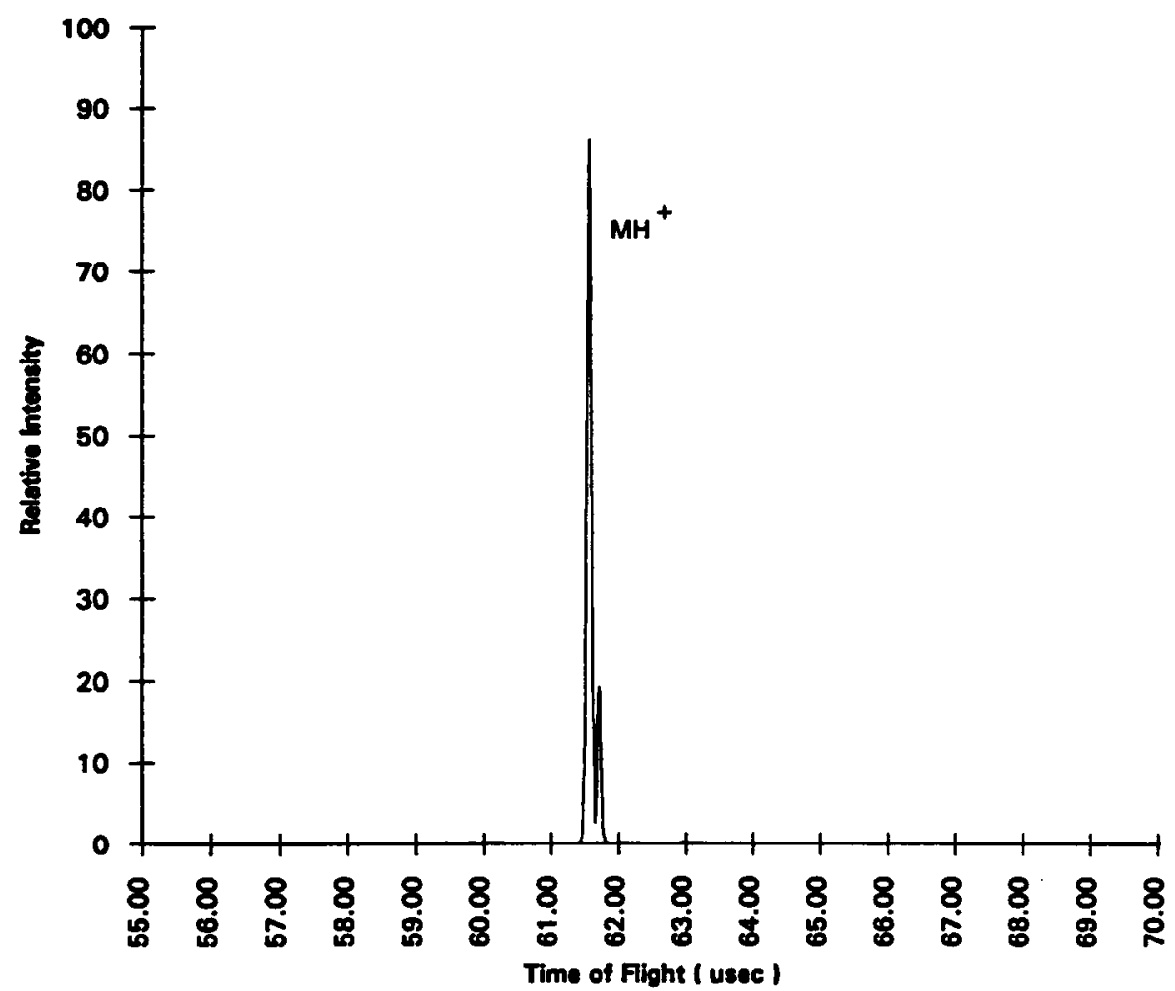

Fig. 14. API mass spectrum of amitriptyline with $V_{\text {push }}=+75 \mathrm{~V}, V_{\text {lens }}=+25 \mathrm{~V}, V_{\mathrm{A}}=-140 \mathrm{~V}, V_{\mathrm{B}}=-70 \mathrm{~V}, V_{\mathrm{rf}}=+530 V_{\mathrm{pp}}$, $V_{\text {extr. }}=-170 \mathrm{~V}, V_{\mathrm{L}}=V_{\mathrm{X} 1}=-1500 \mathrm{~V}, V_{\mathrm{FOC}}=-1250 \mathrm{~V}, V_{\mathrm{X} 2}=-1385 \mathrm{~V}, V_{\mathrm{R} 1}=-100 \mathrm{~V}, V_{\mathrm{R} 2}=+1000 \mathrm{~V}, V_{\text {bias }}=0 \mathrm{~V}$. (Reprinted with permission from ref. 34.)

unit could not be pulsed off before ejection of ions into the RETOF because of the nature of the RF circuit involved. The inability to trigger the r.f. off was shown to result in some peak broadening due to ejection of ions over different phases of the r.f. pulse [28]. Nevertheless, the FWHM width of $m / z$ 279 is $\approx 20 \mathrm{~ns}$ with a resulting resolution of $>1500$ using an ion storage time of $90 \mathrm{~ms}$.

In further work, we investigated the effect of the ion storage time on the sensitivity and resolution in the RETOF. This is shown in Figs. 15(a)-(e) for a continuous ion beam where the mass spectrum of pyridine in the IT-RETOF is monitored as a function of trapping time. The pyridine ions were produced using the API d.c. plasma source as in Fig. 13. Figures 15(a)-(e) show that with the use of a continuous ion beam both the resolution and intensity improve with increased trapping time. For example, in trace (e), the ions are trapped for only a very short period and the result is a spectrum with relatively poor resolution and weak signal intensity similar to that obtained with no trapping under the same conditions. The next spectrum, (d), shows improvement in resolution and signal intensity with only $1 \mathrm{~ms}$ of ion trapping while trace (c), with a moderate trapping time of $90 \mathrm{~ms}$, shows a marked improvement in both of these signal characteristics where a peak of $16 \mathrm{~ns}$ FWHM is observed. Trace (b) shows an extension to long trapping time $(900 \mathrm{~ms})$ and the resultant improvement in resolution and signal intensity where the observed peak is only $10 \mathrm{~ns}$ FWHM. 
Effect of trapping time on resolution and signal intensity

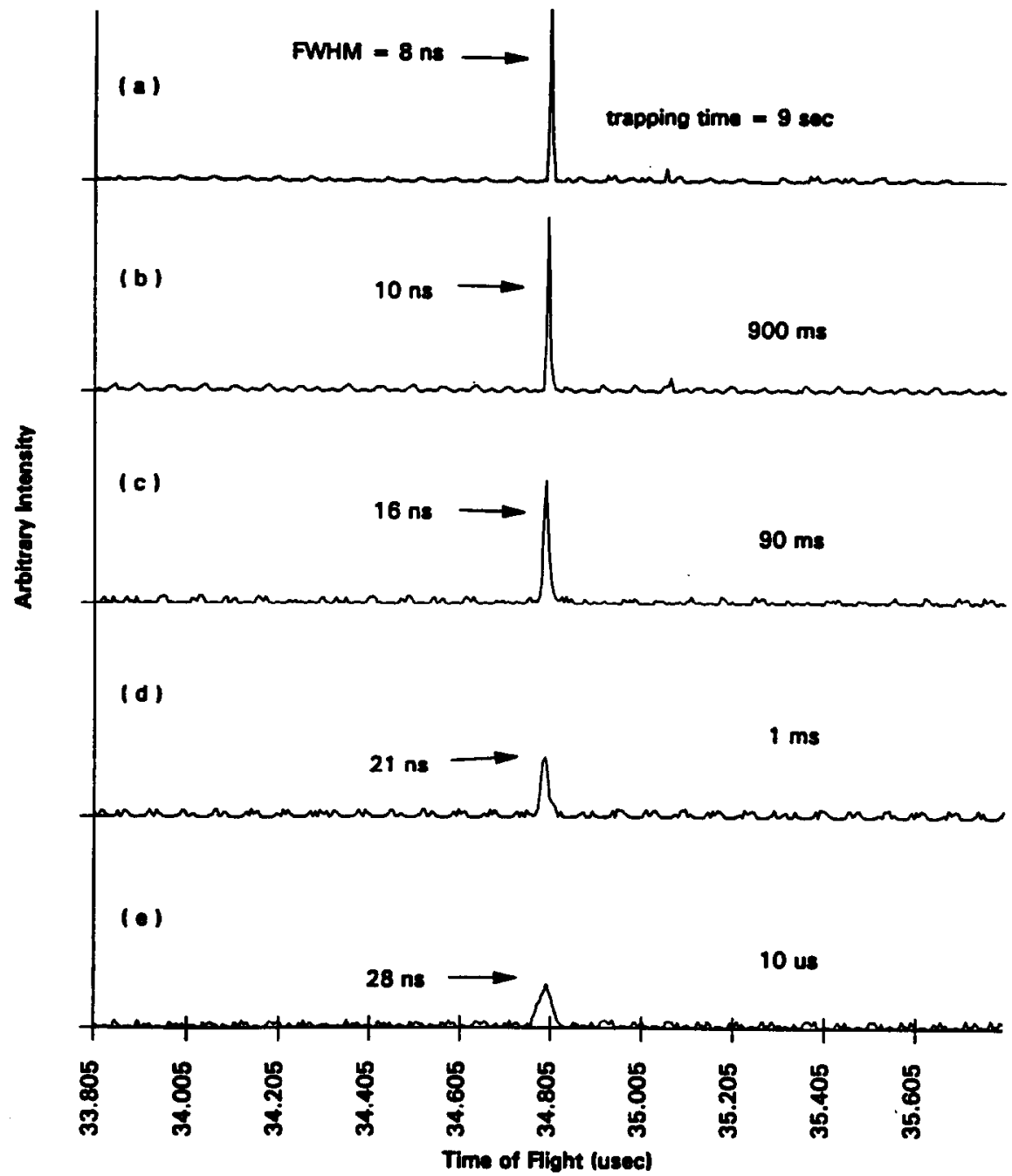

Fig. 15. API mass spectra of pyridine as a function of trapping time: (a) $9 \mathrm{~s}$; (b) $900 \mathrm{~ms}$; (c) $90 \mathrm{~ms}$; (d) $1 \mathrm{~ms}$; (e) $10 \mu \mathrm{s}$. $V_{\text {push }}=+220 \mathrm{~V}$, $V_{\text {lens }}=+170 \mathrm{~V}, V_{\mathrm{A}}=-205 \mathrm{~V}, V_{\mathrm{B}}=-150 \mathrm{~V}, V_{\mathrm{rf}}=+460 V_{\mathrm{pp}}, V_{\text {extr. }}=-225 \mathrm{~V}, V_{\text {bias }}=+345 \mathrm{~V}$, and all other conditions the same as Fig. 13.

Finally, an extremely long trapping time of $9 \mathrm{~s}$ in (a) shows some increase in resolution, $\approx 2100$ (8 ns FWHM), over the previous case with similar signal intensity. The $8 \mathrm{~ns}$ FWHM observed begins to reach the time resolution limit of the PI 9825 signal averager. Thus, an increase in ion trapping time significantly enhances the resolution and signal intensity even up to seconds of storage.

The increase in resolution with extended storage time is presumed to be due to the improved spatial and energy resolution of the ions in the trap using a collisional buffer gas. The extended trapping of ions results in their accumulation in the center of the trap which, upon extraction, yields a more spatially resolved ion packet and thus enhances the spatial resolution of the RETOF. In addition, the large number of collisions with the He buffer gas as a function of storage time collisionally relax 
the translationally hot ions, thus improving the energy component of the resolution. In previous work described earlier [28] using laser-induced REMPI as a means of producing ions inside the trap, the resolution was found to improve rapidly as a function of storage time. In this previous study the resolution reached its optimal value in less than $100 \mu \mathrm{s}$ and little improvement was observed for longer storage times. This indicates that the ions were indeed being rapidly relaxed into the center of the trap. However, these experiments involved ions produced by laser-induced REMPI which resulted in an ion energy distribution of $<0.1 \mathrm{eV}$. In the present study the energy of the ions entering the trap has been measured to be generally around $20 \mathrm{eV}$ although some ions of higher energy may also enter the trap and be trapped. Thus, one might expect a significant enhancement in resolution for these translationally hot externally generated ions over an extended trapping time as indeed is observed. Most of the collisional cooling and thus improvement in resolution, though, does appear to occur within the first several hundred milliseconds.

In order to further study the enhancement of resolution as a function of storage time, the ion beam was pulsed so that an ion beam of a defined duration could be injected into the trap and stored for a variable length of time. In this case ion beams of $100 \mu \mathrm{s}, 500 \mu \mathrm{s}, 1 \mathrm{~ms}$, and $10 \mathrm{~ms}$ were pulsed into the trap and stored from $100 \mu$ s up to several seconds. At storage times above $2 \mathrm{~ms}$ there was essentially no difference between the peak widths obtained using either a continuous or pulsed ion beam. At storage times less than $2 \mathrm{~ms}$ a small difference in peak width could be observed. This difference amounted to $\approx 10 \%$ at $\approx 1.5 \mathrm{~ms}$, where the pulsed ion beam produced a narrower peak width. The most likcly cxplanation for this observation is that, in the case of the continuous ion source, the ions that enter the trap early in the storage cycle will undergo more cooling than the ions that enter towards the end of the cycle. At longer storage times a greater fraction of the ions in the trap will have undergone extensive cooling as compared to shorter storage times. Thus, at longer storage times the ion peak widths of continuous ion sources will be very similar to that of pulsed ion sources. However, at shorter storage times with continuous ion beams, where the number of ions entering the trap at the end of the cycle may be a significant fraction of the ions stored in the trap, one might expect to observe some broadening of the ion peak in the RETOF as compared to a pulsed ion beam. In these experiments such an effect is indeed observed, although the difference between the ion peak width is small even at short storage times and at long storage times there is no measurable difference. In addition, an attempt was made to study the enhancement of resolution as a function of storage time at different He buffer pressures. However, the work reported herein was performed at a He buffer pressure that optimized the signal. A change in the He buffer pressure resulted in a rapid decrease of the signal so that a study of the storage properties as a function of time at various pressures was not possible.

In addition, the signal intensity was also found to increase as a function of storage time. This is due to the integration of the ion signal which occurs over the entire trapping time; hence, for longer trapping time, enhanced ion accumulation occurs and the signal intensity increases. The ion accumulation benefit of the ion trap has a limit in its ability to enhance signal intensity, for at some point saturation of the trap with resulting space charge losses will be observed.

Reduced pressure ionization. External ion injection into the IT-RETOF was also investigated for other ion sources. In particular, a low pressure glow discharge was produced between the two skimmers in the differentially pumped orifice region which was used to ionize organic molecules sampled from the API [35]. The results obtained using this low pressure glow with detection by the IT-RETOF are shown in Fig. 16 for detection of dibenzothiophene sample from liquid injection into an atmospheric pressure source. This figure illustrates an important advantage of the IT-RETOF 


\section{Reduced Presaure Clow Discharge lonization of Dibenzothiophens, MW} 184, 5 me trapping time.

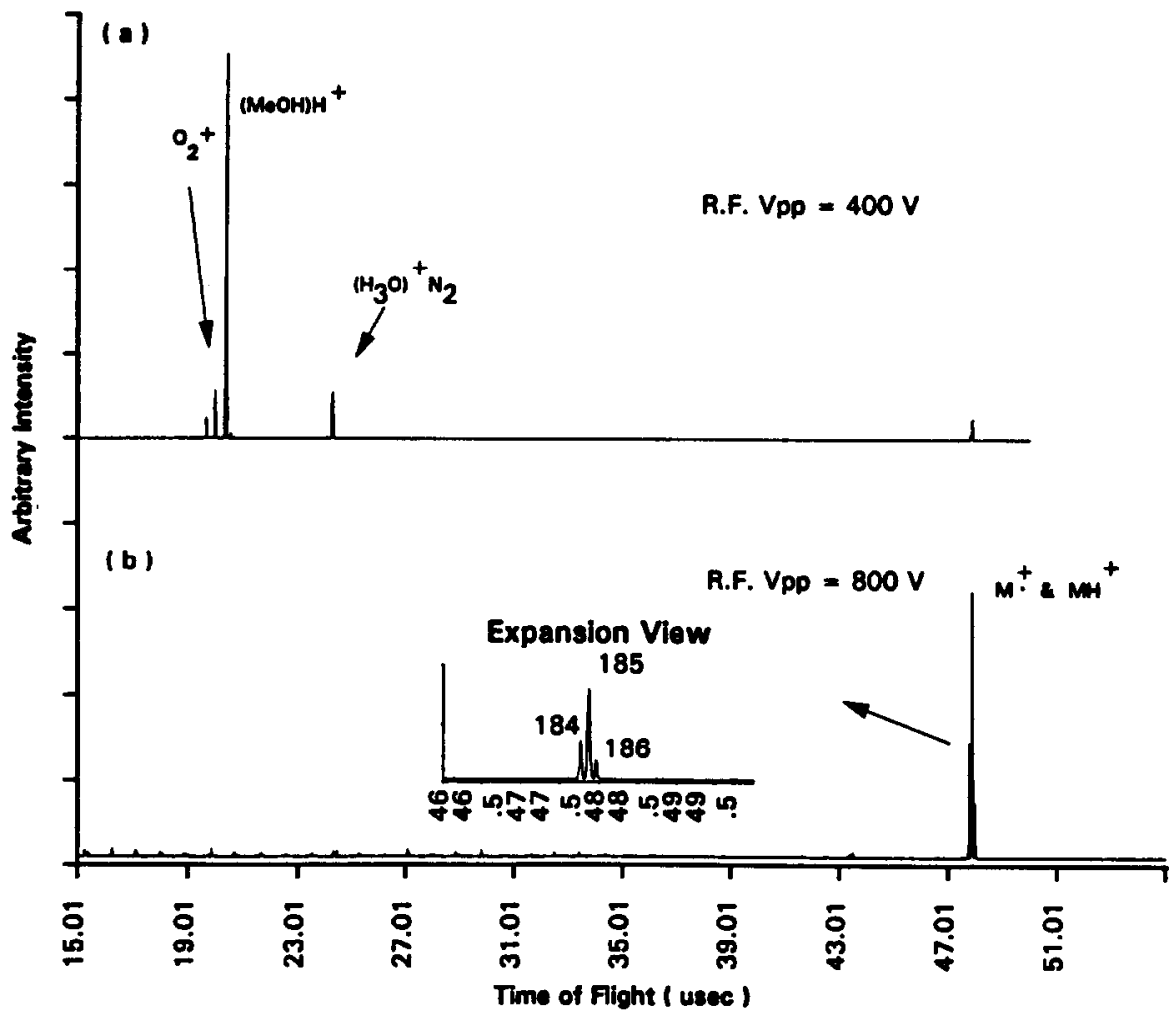

Fig. 16. Reduced pressure glow discharge ionization mass spectra of dibenzothiophene. (a) $V_{\text {r.f. }}=400 \mathrm{~V}_{\mathrm{pp}} ;(\mathrm{b}) V_{\mathrm{r} . \mathrm{f}}=800 \mathrm{~V}_{\mathrm{pp}}$, and $V_{\mathrm{A}}=+100 \mathrm{~V}, V_{\mathrm{B}}=+200 \mathrm{~V}, V_{\text {extr. }}=-160 \mathrm{~V}, V_{\mathrm{L}}=V_{\mathrm{X} 1}=-1500 \mathrm{~V}, V_{\mathrm{FOC}}=0 \mathrm{~V}, V_{\mathrm{X} 2}=-1385 \mathrm{~V}, V_{\mathrm{R} 1}=+150 \mathrm{~V}, V_{\mathrm{R} 2}=+1000 \mathrm{~V}$ (Reprinted with permission from ref. 34.)

in its ability to eliminate background ions based upon the selected trapping conditions. This is a potential problem in the presence of large background ion peaks which may saturate the trap or the detector, especially when attempting to amplify the smaller signal peaks. Figures 16(a) and (b) demonstrate the effect of changing the r.f. potential applied to the ring electrode of the ion trap on the observed spectrum and its ability to eliminate background ions. These spectra are of dibenzothiophene in $10^{-4} \mathrm{M}$ methanol solution, ionized via the low pressure glow discharge in He. In Fig. 16(a), the r.f. applied was $400 \mathrm{~V}_{\mathrm{pp}}$ and the observed spectrum is dominated by the background ion peaks with a trace analyte peak observed. However, by increasing the applied r.f. to $800 \mathrm{~V}_{\mathrm{pp}}$ the trapped $m / z$ range is shifted to higher $m / z$, eliminating the background ion peaks from the observed spectrum as shown in Fig. 16(b). Because the trap under this condition was not saturated by the background ion peaks, more of the analyte was stored and accumulated, resulting in the enhancement of analyte signal intensity. Thus, the r.f. potential applied to the ring electrode determines the $m / z$ range that can be trapped. By varying the r.f. amplitude, the $m / z$ range trapped can be selected to eliminate low mass background.

An important problem encountered in interfacing an atmospheric pressure generated ion beam to the ion trap is selecting the correct parameters to optimize the kinetic energy of the beam. The ion trap device is most efficient in trapping ions 
of relatively low energy, i.e. $0-20 \mathrm{eV}$ [36-38]. It was found that the pressure in the differentially pumped region is particularly important in the ion transmission. In the API experiments performed herein the first skimmer orifice is $275 \mu \mathrm{m}$ and the second skimmer orifice is $325 \mu \mathrm{m}$. This results in a pressure of $\approx 1.5$ Torr in the differentially pumped region. Under these conditions the ion beam energy is very dependent on $V_{\text {lens }}$, i.e. the focussing ring electrode voltage. The signal was found to be most intense with a $V_{\text {lens }} \approx 30 \mathrm{~V}$.

The key parameters deciding the ion energy and transmission efficiency from the ionization chamber through the dual orifice interface were $V_{\text {push }}$ and $V_{\text {lens }}$. $V_{\text {push }}$ aided in pushing the ions from the API chamber into the dual orifice interface. Although the pressure differential was the main factor influencing the drawing of ions into and through the interface region, a $V_{\text {push }}$ of +70 to $+150 \mathrm{~V}$ was experimentally determined to significantly enhance the ion current traversing the interface region. This parameter was optimized with each individual experimental run. Limitations were that $V_{\text {push }}<+70 \mathrm{~V}$ provided no significant increase in ion throughput and $V_{\text {push }}>+150 \mathrm{~V}$ caused a reduced pressure glow discharge to form in the differentially pumped interface region. $V_{\text {lens }}$ served to condense the ion beam emanating from the first skimmer and focus it upon the second skimmer's orifice. This parameter was also found to be a critical factor influencing the energy of the ions entering the pre-trap Einzel lens. The $V_{\text {lens }}$ typically was run at +10 to $+35 \mathrm{~V}$. A setting within this range was found to provide effective ion beam focussing without imparting too much energy to the ions. Greater $V_{\text {lens }}$ settings create an ion beam with too much energy to effectively trap ions without a d.c. bias being applied to the trap.

In further work, it was found that high energy ions could also be stored within the trap. If the first skimmer was changed to $350 \mu \mathrm{m}$, the pressure in the differentially pumped region was typically 5-10 Torr, under our operating conditions. Under these conditions the ion transmission efficiency is very low and only ions with higher energy were sampled into the mass spectrometer region. Indeed, a high voltage on $V_{\text {lens }}$ was needed to efficiently focus ions into the second skimmer. In addition, the He flow rate in the API chamber was found to be critical, where a high He flow rate enhanced the resulting transmission of ions but also appeared to increase the energy of the ions. An ion energy measurement in the mass spectrometer was performed using a blocking grid and the ion energy was found to be as high as several hundred electronvolts. However, using the technique described in the experimental section whereby high energy ions can be slowed down and trapped by using a positive voltage bias on the ring electrode and both end caps, ions of up to $300 \mathrm{eV}$ could be injected from an external source and trapped.

ESI. The results of interfacing this IT-RETOF mass spectrometer with an ESI source are demonstrated in Figs. 17-20. Figure 17 shows the ESI mass spectrum of singly and doubly charged Arginine molecular ion injected into the IT-RETOF using $1.2 \times 10^{-6} \mathrm{M}$ Arginine dissolved in $\mathrm{MeOH} /$ $\mathrm{H}_{2} \mathrm{O} / \mathrm{HOAc}(80 / 20 / 5)$ mixed solvent. The production and transmission of ions to the trap were performed as described before in the experimental section. The spectrum obtained was a single waveform spectrum with a storage time of $931 \mathrm{~ms}$. Thus, only $1 \mathrm{~s}$ is needed to acquire this spectrum with $S / N$ of the molecular ion peak greater than 20 . The actual Arginine sample consumed is $200 \mathrm{fmol}$. This clearly demonstrates the ability of the IT-RETOF to detect trace transient species or rapidly eluting species. The FWHM of the $\mathrm{MH}^{+}$ peak is $10 \mathrm{~ns}$ which corresponds to a mass resolution of $\approx 2700$. Because the production of ions of different charges is a statistically distributed process, the relative intensity of singly charged vs. doubly charged molecular ion varied from one single waveform to another. However, as shown in Fig. 18, the spectrum obtained after being averaged 50 times showed a reproducible signal intensity pattern of both charged ions under the same experimental conditions. The FWHM of the $\mathrm{MH}^{+}$ ion of Arginine in Fig. 18 is $16 \mathrm{~ns}$ which corre- 


\section{Electrospray lonization of Arginine, MW 174, 931 ms trapping timo, single wavoform spectrum}

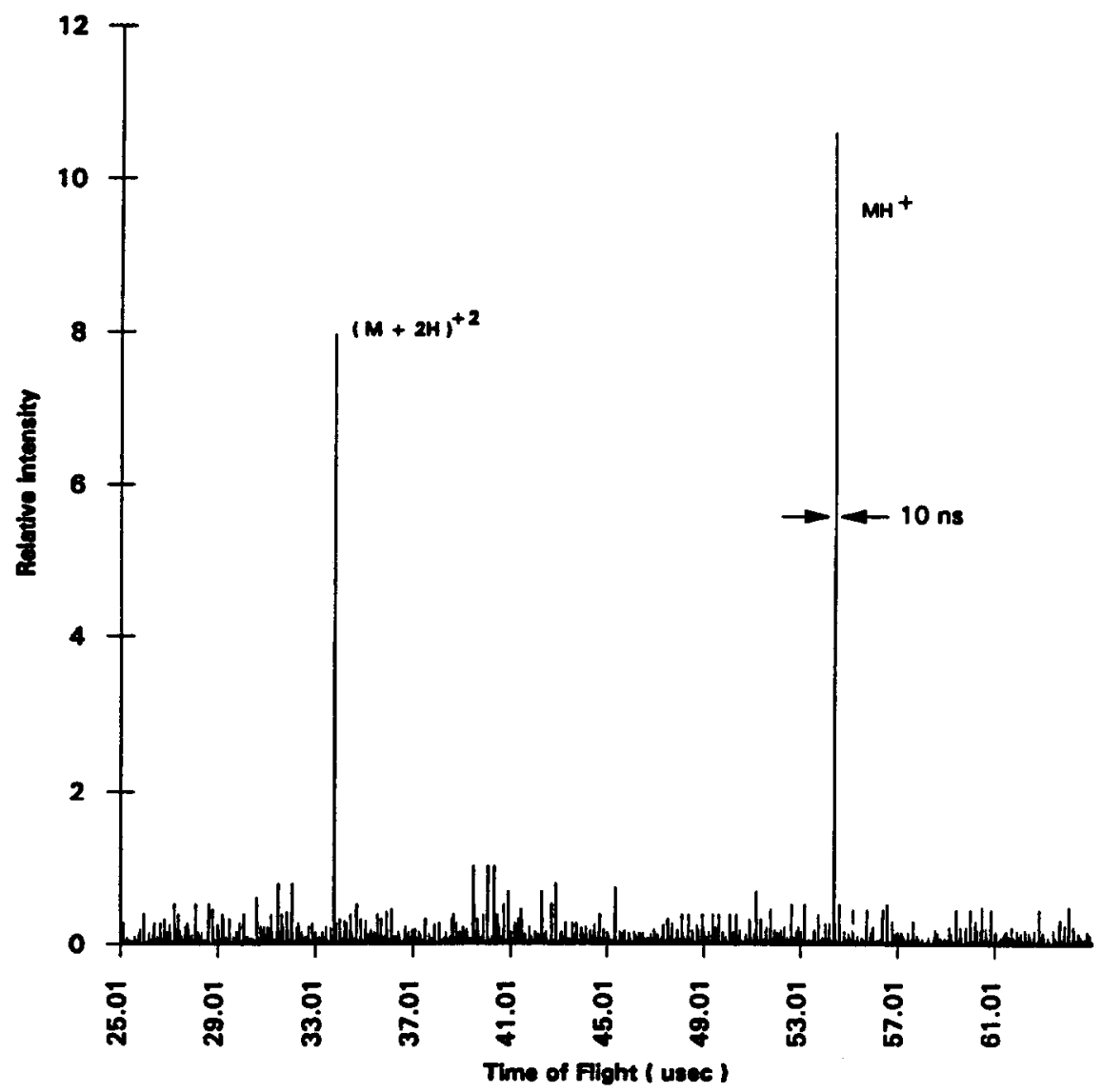

Fig. 17. ESI single waveform spectrum of Arginine with $V_{\text {r.f. }}=500 \mathrm{~V}_{\mathrm{pp}}, V_{\text {cap }}=+50 \mathrm{~V}, V_{\text {skimmer }}=+32 \mathrm{~V}, V_{\text {lens }}=+90 \mathrm{~V}, V_{\mathrm{A}}=-17 \mathrm{~V}$, $V_{\mathrm{B}}=-65 \mathrm{~V}, V_{\text {extr. }}=-400 \mathrm{~V}, V_{\mathrm{L}}=V_{\mathrm{XI}}=-1500 \mathrm{~V}, V_{\mathrm{FOC}}=-1250 \mathrm{~V}, V_{\mathrm{X} 2}=-1385 \mathrm{~V}, V_{\mathrm{R} 1}=-197 \mathrm{~V}, V_{\mathrm{R} 2}=+410 \mathrm{~V}$

sponds to a resolution of $>1700$. The degradation of resolution in the averaged spectrum was the result of using the modified EAI quadrupole r.f. power supply which could not be pulsed off. The actual sample consumed in obtaining Fig. 18 was 1 pmol. A voltage of $530 \mathrm{~V}_{\mathrm{pp}}$ was used at a frequency of $1.1 \mathrm{MHz}$ in obtaining the above two spectra.

The ability to obtain enhanced resolution at higher molecular weight through the use of ion storage is demonstrated in Fig. 19 for the molecular ion region of bradykinin. Figure 19 shows the expansion region of the $\mathrm{MH}^{+}$peak of bradykinin obtained using ESI produced ions from
$2.3 \times 10^{-6} \mathrm{M}$ bradykinin dissolved in $\mathrm{MeOH} /$ $\mathrm{H}_{2} \mathrm{O} / \mathrm{HOAc}(80 / 20 / 5(\mathrm{v} / \mathrm{v}))$ solvent injected into the IT-RETOF. The spectrum obtained was a 400 waveform average using a storage time of $931 \mathrm{~ms}$ per cycle. The r.f. voltage used was 1975 $V_{p p}$ and only 12 pmol of sample was used in obtaining this spectrum. The peak of highest intensity observed in Fig. 5 is the bradykinin $\mathrm{MH}^{+}$peak at $m / z 1061$. The FWHM of this peak is only $20 \mathrm{~ns}$ which corresponds to a mass resolution of $\approx 3300$. This spectrum clearly shows the completely resolved ${ }^{13} \mathrm{C}$ isotope peaks with $\mathrm{m} / \mathrm{z}$ of 1062 and 1063. The ions with $m / z$ of 1060 and 1059 were also observed. These two ions are the result of loss of 

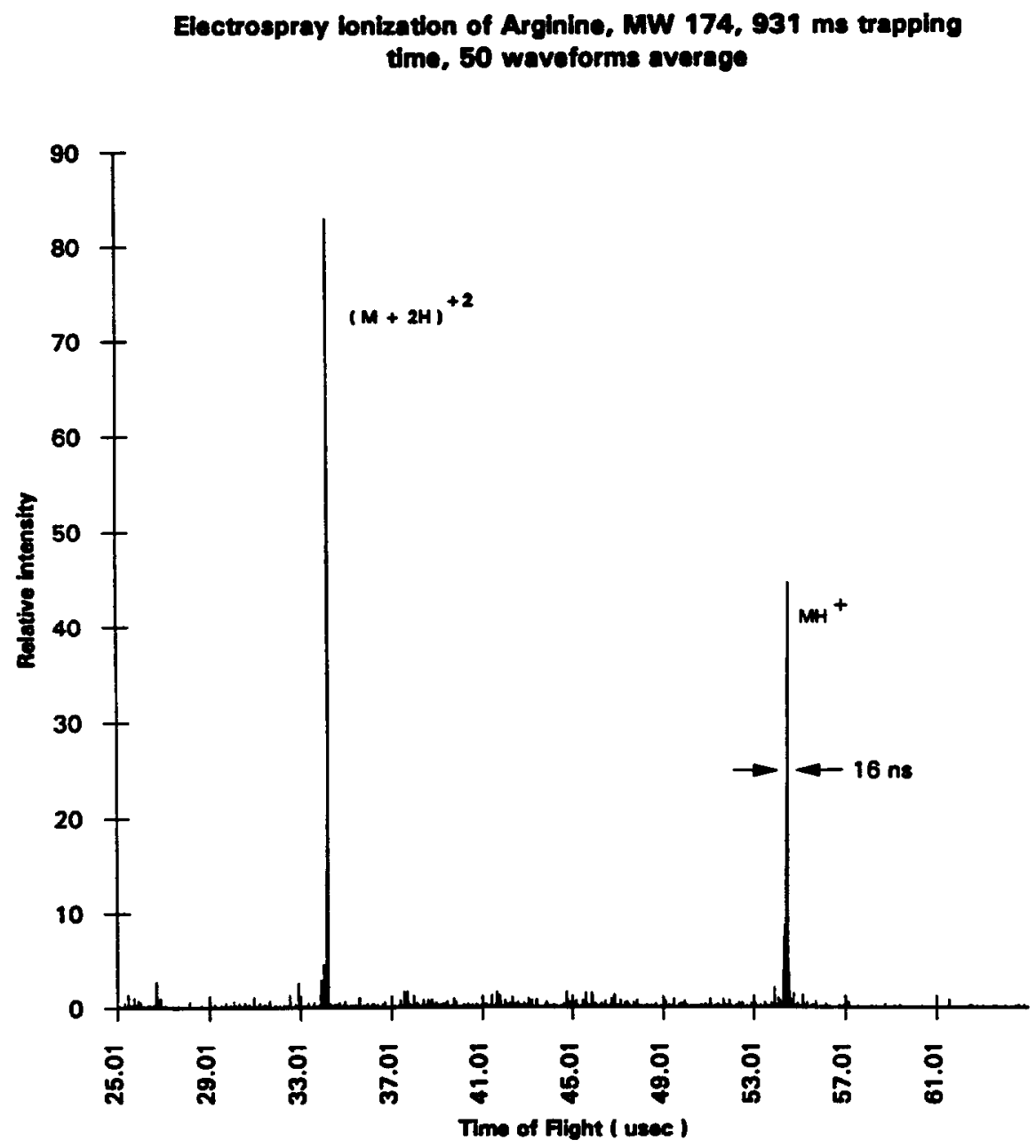

Fig. 18. Fifty waveform average of ESI Arginine spectrum. All conditions same as those of Fig. 17.

one and two protons respectively. The appearance of these ions is more prominent when higher r.f. voltage was employed suggesting that the loss of $\mathrm{H}$ atoms most probably occurred from collisions inside the trap.

In Figs. 20 and 21 are shown ESI-IT-RETOF mass spectra of Gramicidin S illustrating the use of different storage time on the $S / N$ quality of the IT-RETOF spectra observed. The spectra were obtained using $10^{-6} \mathrm{M}$ Gramicidin $\mathrm{S}$ dissolved in $\mathrm{MeOH} / \mathrm{H}_{2} \mathrm{O} / \mathrm{HOAc}(80 / 20 / 5)$ mixed solvent. In Fig. 20, the spectrum was obtained using a $3.1 \mathrm{~ms}$ storage time and was averaged 50 times. This corresponds to a total analysis time of only $1.5 \mathrm{~s}$. The
Gramicidin S sample actually consumed in obtaining this spectrum is $250 \mathrm{fmol}$. The major pcak observed in this spectrum is the singly charged Gramicidin S molecular ion with a $m / z$ of 1142 . The doubly charged molecular ion with a $\mathrm{m} / \mathrm{z}$ of 571.5 was also observed as the second largest peak in the spectrum. A r.f. voltage of $2200 \mathrm{~V}_{\mathrm{pp}}$ was used to obtain this spectrum. Under the same conditions, another spectrum was obtained using a $931 \mathrm{~ms}$ storage time and was averaged 50 times as shown in Fig. 21. The $S / N$ ratio is greatly improved in this spectrum because of the ability of the ion trap to accumulate the ions through longer trapping time. This capability is especially 


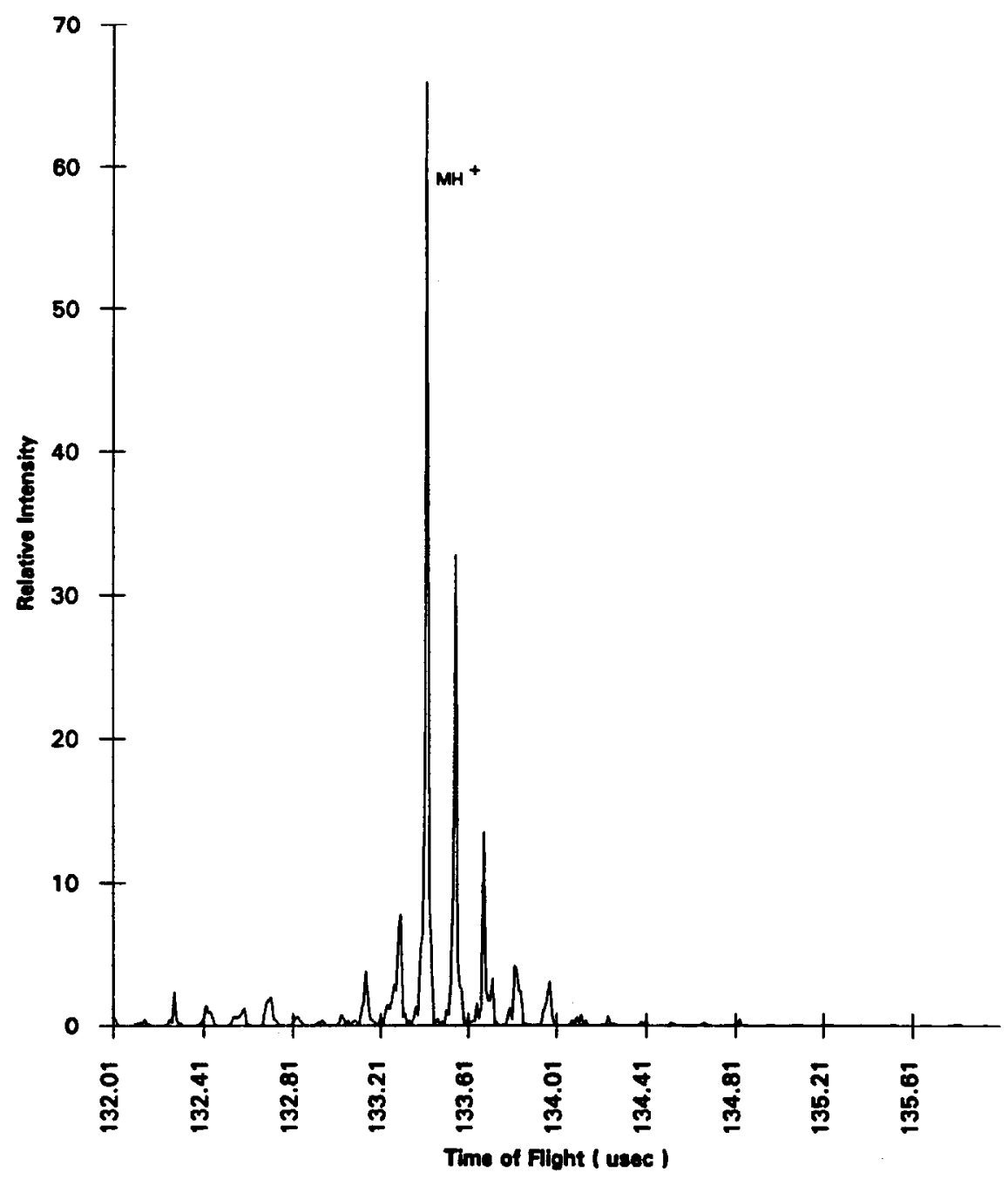

Fig. 19. ESI spectrum of Bradykinin showing expanded view of molecular ion peak with $V_{\text {r.f. }}=1975 V_{\text {pp }}, V_{\text {cap }}=+50 \mathrm{~V}$, $V_{\text {skimmer }}=+10 \mathrm{~V}, V_{\text {lens }}=+195 \mathrm{~V}, V_{\mathrm{A}}=-92 \mathrm{~V}, V_{\mathrm{B}}=-70 \mathrm{~V}, V_{\text {extr, }}=-330 \mathrm{~V}$. All other conditions same as those of Fig. 17.

prominent when interfaced to an ESI source as opposed to a plasma source where the ion trap is easily saturated because of the high ion current $(1-10 \mathrm{~mA})$ produced in the ionization source. Generally, the ion current measured in the ion trap region is usually less than a few picoamperes in our current experimental setup. This results in the reduction of space charge effects inside the ion trap. Thus, the resolution of all the spectra obtained using the ESI source is generally improved over that obtained from the plasma source. The FWHM of Gramicidin S doubly charged molecular ion peak in Figs. 20 and 21 is $\approx 35 \mathrm{~ns}$ which corresponds to a mass resolution of $\approx 1900$. The amounts of Gramicidin $S$ actually consumed in these two spectra were $240 \mathrm{fmol}$ and $7.2 \mathrm{pmol}$ respectively.

The present limit for trapping externally generated ions via electrospray is $\approx 3000$. Nevertheless this has allowed us to trap and detect large 


\section{Electrospray ionlzation of Gramicidin S, MW 1141, 3.1 ms}

trapping time, 50 waveforms average

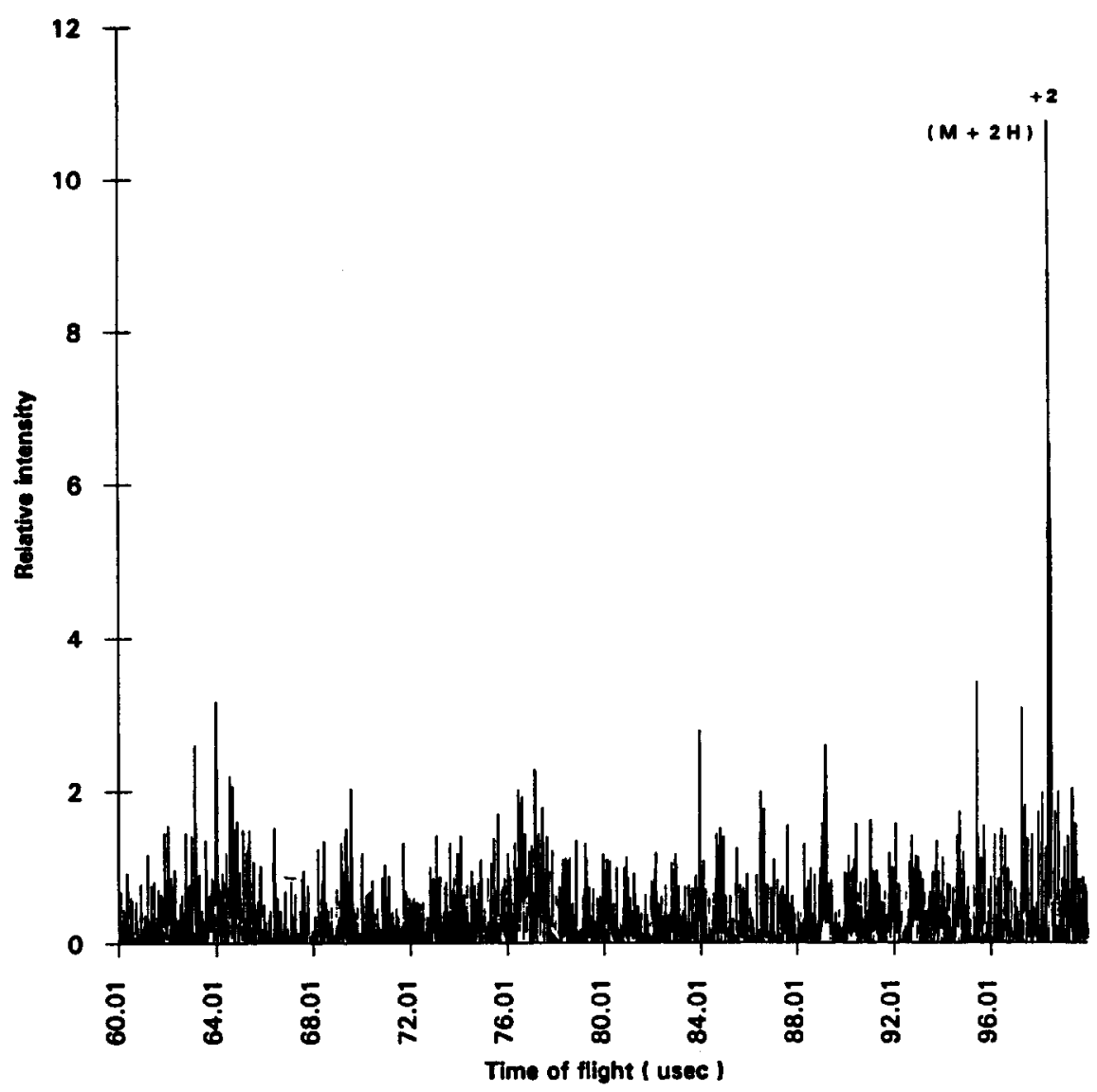

Fig. 20. ESI spectrum of Gramicidin S showing the doubly charged molecular ion peak with $V_{\text {r.f. }}=2200 \mathrm{~V}_{\text {pp }}, V_{\text {cap }}=+50 \mathrm{~V}$, $V_{\text {skimmer }}=+10 \mathrm{~V}, V_{\text {lens }}=+195 \mathrm{~V}, V_{\mathrm{A}}=-92 \mathrm{~V}, V_{\mathrm{B}}=-70 \mathrm{~V}, V_{\text {extr. }}=-330 \mathrm{~V}$. All other conditions same as those of Fig. 17.

multiply-charged peptides including Melittin $(\mathrm{m} / \mathrm{z}$ 2845), bovine cytochrome $C(m / z$ 12327), horse heart myoglobin $(m / z 16950)$ and bovine albumin $(m / z 66266)$. This is shown for the case of cytochrome $\mathrm{C}$ in Fig. 22 where multiply charged ions from $(\mathrm{M}+11 \mathrm{H})^{11+}$ to $(\mathrm{M}+20 \mathrm{H})^{20+}$ are observed. This spectrum was obtained using $4.8 \times 10^{-7} \mathrm{M}$ cytochrome $\mathrm{C}$ dissolved in $\mathrm{MeOH} / \mathrm{H}_{2} \mathrm{O} / \mathrm{HOAc}$ $(50 / 50 / 2.5)$ mixed solvent. The storage time used here was $931 \mathrm{~ms}$ and the spectrum was averaged 200 times. The amount of cytochrome $\mathrm{C}$ sample actually consumed in obtaining this spectrum is $400 \mathrm{fmol}$. The maximum r.f. voltage of $2200 \mathrm{~V}$ was used in trapping these externally generated ions. In order to extend the mass range a higher r.f. voltage will yet be required.

An important potential advantage of the IT-RETOF is the duty cycle of the device. The actual pulse out time of the device is $\approx 2 \mu \mathrm{s}$. Thus, if one uses a storage time of $>10 \mathrm{~ms}$ per cycle for sampling a continuous ion beam, the duty cycle approaches nearly $100 \%$. Other methods have been used to sample continuous ion beams into TOF such as beam modulation and pulsed extraction. In the beam modulation technique an ion beam is swept past a slit to obtain time resolution in the TOF device. In previous work with an API beam, this method was shown to provide excellent 


\section{Electrospray lonization of Gramicidin S, MW 1141. $931 \mathrm{~ms}$ trapping time, 50 waveforms average}

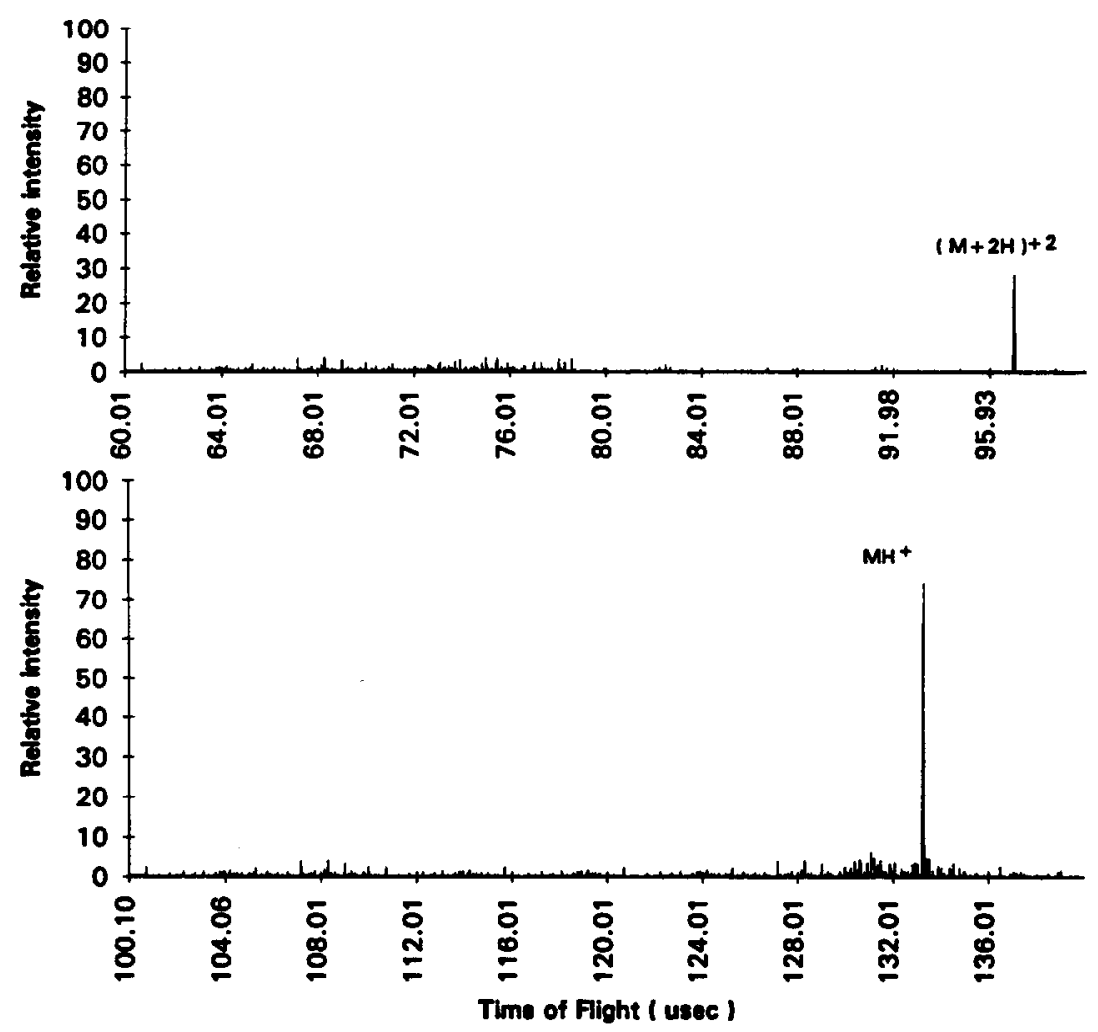

Fig. 21. ESI spectrum of Gramicidin $\mathrm{S}$ showing both the singly and doubly charged molecular ion peaks. All conditions are same as those of Fig. 19.

resolution in a linear TOF-MS [39]; however, the sensitivity was rather limited due to the poor duty cycle. It is estimated that even with a beam modulation rate of $10 \mathrm{kHz}$, a maximum duty cycle of $\approx 0.02 \%$ can be achieved. An alternative method for interfacing a continuous ion beam to the TOF is the pulsed extraction technique. In this method an ion beam is transmitted between the acceleration plates of the TOF and then rapidly pulsed out with an extraction pulse into the TOF drift tube which is transverse to the ion beam. The duty cycle is limited by the length of the extraction plates and velocity of the ion beam, but has been estimated to be as high as $\approx 2.5 \%$ based upon a repetition rate of $10 \mathrm{kHz}$ [40]. The duty cycle of these two techniques will decrease when detecting ions with larger $m / z$ where a lower repetition rate must be used.
An advantage of the ion trap is that it can achieve an excellent duty cycle for continuous ion beams independent of repctition rate. As shown in this work, a trapping time of $100 \mathrm{~ms}$ can be used to enhance the signal and resolution of ions generated by an API source. Under these conditions, a pulseout rate of only $10 \mathrm{~Hz}$ is required, which can easily be processed even with relatively modest electronics and software. When fast spectrum acquisition must be performed, such as applications in GC or LC when detecting rapidly eluting species or monitoring transient species, a $100 \mu \mathrm{s}$ trapping time and a pulse-out rate of $10 \mathrm{KHz}$ can be used to obtain the same $100 \%$ duty cycle. In contrast, the beam modulation and pulsed extraction methods require a high extraction rate $(10 \mathrm{kHz})$ to achieve a reasonable duty cycle. Although this is 


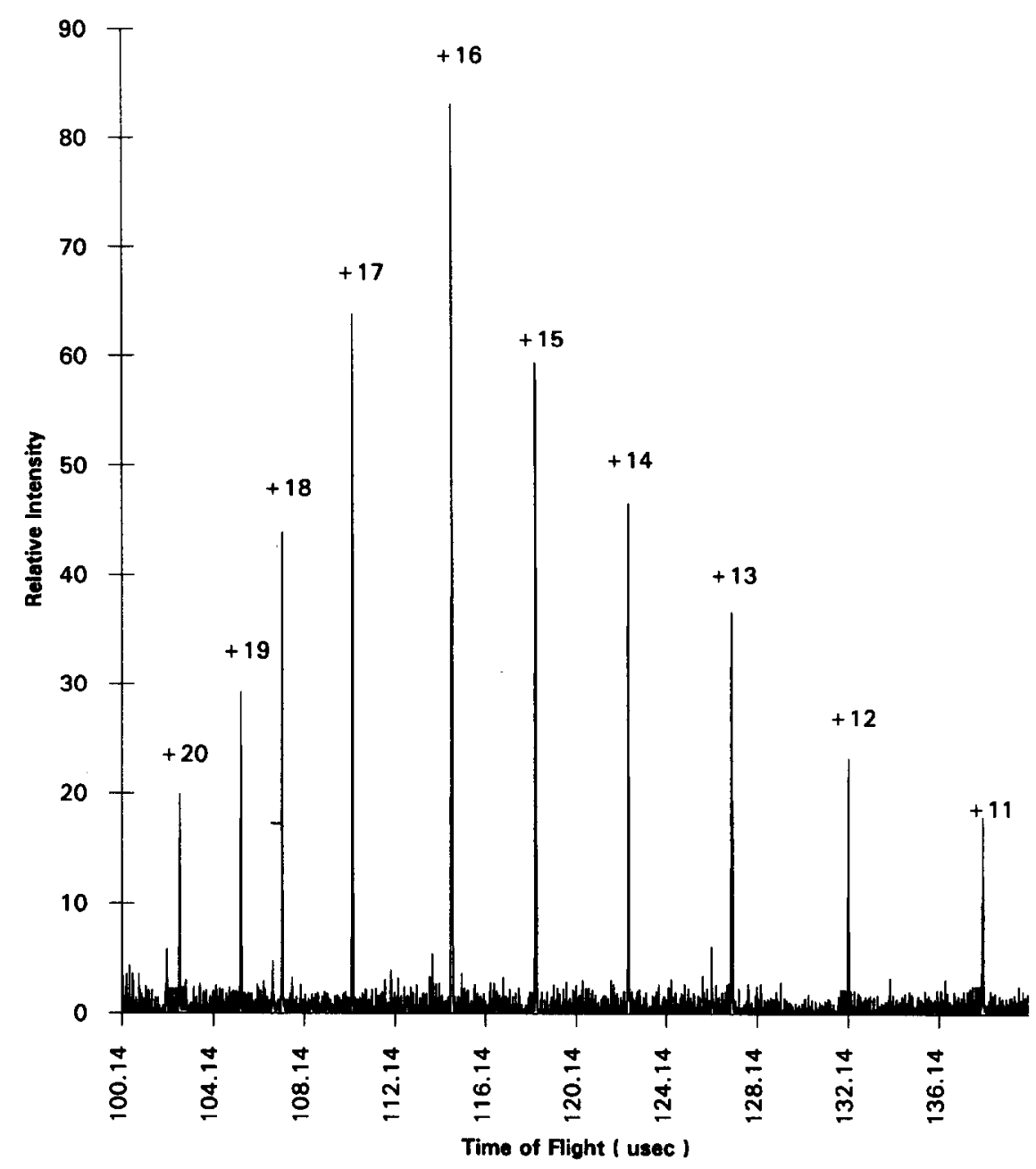

Fig. 22. ESI of cytochrome C showing multiply charged peaks with $V_{\text {r.f. }}=2200 \mathrm{~V}_{\mathrm{pp}}, V_{\text {cap }}=+100 \mathrm{~V} . V_{\text {skimmer }}=+30 \mathrm{~V}, V_{\text {lens }}=+160 \mathrm{~V}$, and $V_{\text {extr. }}=-330 \mathrm{~V}$. All other conditions same as those of Fig. 17.

possible to process with modern digitizers and software, the use of the low repetition rate provided by the IT-RETOF greatly simplifies data collection and processing.

There are other potential advantages of the IT-RETOF over pulsed extraction methods without storage. Simulations performed on the sIMION program show that orthogonal extraction of ions becomes difficult above $50 \mathrm{eV}$ of energy where the ions can not be easily turned around and trans- mitted down the flight tube. In comparison, the IT-RETOF is capable of slowing down and trapping ions of high energy with resulting high resolution due to the storage properties of the trap. Also, the orthogonal pulsed extraction method will be limited in the mass range that will be observed due to the transverse velocity component of the ion beam. However, the mass storage range of the IT-RETOF is determined by the voltage and frequency applied to the trap and can be made 
extremely large in conjunction with the use of a buffer gas in the trap. In recent simulations [28] it has been shown that a storage range of several hundred thousand atomic mass units should be possible under appropriate conditions. In the present work the ability to trap externally generated ions is limited to $m / z<3000$ by the voltage range of the r.f. power supplies available. However, storage of ions over a relatively large mass range has been demonstrated in the trap $[41,42]$. The subsequent pulse-out of the ions from the trap into the RETOF occurs on-axis so that any further energy difference in the kinetic energy of ions of different masses in the extraction process is no longer important. In addition, a further possible advantage of the IT-RETOF in these experiments that has not been demonstrated herein is the possibility for MS-MS studies in the trap and for the studies of long-lived metastable decay in the IT-RETOF combination.

A measurement of the limit of detection attainable for liquid injection-nebulization into the plasma source was examined using pyridine dissolved in methanol solution. An initial solution of $6.55 \times 10^{-6} \mathrm{M}$ was prepared and successive dilutions were made down to $6.55 \times 10^{-10} \mathrm{M}$. These samples were run as detailed in this work, but with the storage time optimized for each concentration. A lower limit of detection of 2-3 fmol was determined using a $S / N=3$ as our limit of detection criterion. Similar limits of detection were observed for liquid injection into the ESI source using Arginine, Leucine-Alanine and Gramicidin $\mathrm{S}$ dissolved separately in $\mathrm{MeOH} / \mathrm{H}_{2} \mathrm{O} / \mathrm{HOAc}$ $(80 / 20 / 5 \quad(v / v))$ solvent. Initial solutions of $7 \times 10^{-5} \mathrm{M}$ were prepared and successive dilutions were made down to $6.55 \times 10^{-8} \mathrm{M}$. These samples were run as detailed previously but with the conditions optimized for each analyte and concentration. A lower limit of detection of 35, 20 and $80 \mathrm{fmol}$ respectively were determined using $S / N=3$ as our limit of detection criterion. Later work on ESI incorporated a $40 \mathrm{~mm}$ triple microchannel plate detector in our IT-RETOF mass spectrometer where limits of detection of $4.5,0.7$, and $0.5 \mathrm{fmol}$ were achieved for Gramicidin S, Arginine, and Leucine-Alanine respectively. Future work will involve improving the ion transmission effeciency in the interface region, which should allow even lower detection limits.

In conclusion, the capabilities of an IT-RETOF MS combination have been demonstrated for detection of ions generated by liquid injection into plasma source atmospheric pressure ionization mass spectrometry and detection of ions generated from an ESI ionization source. The ion trap has been shown to be an effective means of storing externally generated ions for up to $10 \mathrm{~s}$, prior to mass analysis in the RETOF MS. The IT-RETOF can provide nearly $100 \%$ duty cycle in converting a continuous ion beam into a pulsed source for TOF, which will be important for interfacing to chromatographic applications. It is also shown that the storage capabilities of the device provide enhanced resolution and sensitivity as the storage time is increased. The detection limit of the device was also demonstrated with liquid injection techniques in the plasma source and found to be in the low femtomole range, while similar low femtomole detection limits were also observed for ESI of peptides. In addition, the r.f. voltage was shown to be an effective means of eliminating low mass background peaks from the trap and, thus, from the TOF mass spectrum obtained.

\section{Acknowledgements}

We thank Bruce Thomson of Sciex for the loan of an electrospray source. This work received partial support from the National Science Foundation under grant BIR-9223677.

\section{References}

1 W.C. Wiley and I.H. McLaren, Rev. Sci. Instrum., 26 (1955) 1150.

2 D. Price and J.E. Williams (Eds.), Time-of-Flight Mass Spectrometry, Pergamon, Oxford, 1969.

3 D.M. Lubman and M.N. Kronick, Anal. Chem., 54 (1982) 660 . 
4 J.M.B. Bakker, J. Phys. E., 6 (1973) 785.

5 D.M. Lubman and R.M. Jordan, Rev. Sci. Instrum., 56 (1985) 373.

6 R.B. Opsal, K.G. Owens and J.P. Reilly, Anal. Chem., 57 (1985) 1884.

7 B.A. Mamyrin, V.I. Karataev, D.V. Shmikk and V.A. Zagulin, Sov. Phys.-JETP, 37 (1973) 45.

8 U. Boesl, H.J. Neusser, R. Weinkauf and E.W. Schlag, J. Phys. Chem, 86 (1982) 4851.

9 R. Frey, G. Weiss, H. Kaminsky and E.W. Schlag, Z. Naturforsch. Teil A, 40 (1985) 1349.

10 D.M. Lubman, W.E. Bell and M.N. Kronick, Anal. Chem., 55 (1983) 1437.

11 T. Bergmann, T.P. Martin and H. Schaber, Rev. Sci. Instrum., 60 (1989) 347; 60 (1982) 792.

12 R. Grix, R. Kutscher, G.Li, U. Gruner and H. Wollnik, Rapid Commun. Mass Spectrom., 2 (1988) 83.

13 U. Boesl, R. Weinkauf and E.W. Schlag, Int. J. Mass Spectrom. Ion Processes, 112 (1992) 121.

14 R.C. Beavis and B.T. Chait, Rapid Commun. Mass Spectrom., 3 (1989) 233.

15 J.G. Boyle, C.M. Whitehouse and J.B. Fenn, Rapid Commun. Mass Spectrom, 5 (1991) 400.

16 R.J. Cotter, Anal. Chem. A, 64 (1992) 1027.

17 X. Tang, R. Beavis, W. Ens, F. Lafortune, B. Schueler and K.G. Standing, Int. J. Mass Spectrom. Ion Processes, 85 (1988) 43.

18 W. Paul, Angew. Chem., Int. Ed.Engl., 29 (1990) 739.

19 B.D. Nourse and R.G. Cooks, Anal. Chim. Acta, 228 (1990) 1.

20 R.G. Cooks and R.E. Kaiser, Jr. Acc. Chem. Res., 23 (1990) 213.

21 J.S. Brodbelt-Lustig and R.G. Cooks, Spectra, 11 (1988) 30.

22 J.N. Louris, J.S. Brodbelt and R.G. Cooks, Int. J. Mass Spectrom. Ion Processes, 75 (1987) 345.

23 G. Van Berkel, G. Glish and S. McLuckey, Anal. Chem., 62 (1988) 1284.

24 R. Cooks, G. Glish, S. McLuckey and R. Kaiser, C \& E News, March 25 (1991) 26.
25 R. March and R. Hughes, Quadrupole Storage Mass Spectrometry, Wiley, New York, 1989.

26 R. Cooks, J. Williams, K. Cox, K. Kaiser and J. Schwartz, Rapid Commun. Mass Spectrom., 5 (1991) 327.

27 D.E. Goeringer, S.A. McLuckey and G.L. Glish, Proc. 39th ASMS Conference on Mass Spectrometry and Allied Topics 1991, Nashville, TN, p. 532.

28 S.M. Michael, M.Chien and D.M. Lubman, Rev. Sci. Instrum., 63 (1992) 4277.

29 C.Ma, H. Lee and D.M. Lubman, Appl. Spectrosc., 46 (1992) 1769.

30 J. Zhao, J. Zhu and D.M. Lubman, Anal. Chem., 64 (1992) 1426.

31 R.C. Willoughby and R.F. Browner, Anal. Chem., 56 (1984) 2626.

32 I. Sofer, J. Zhu, H.S. Lee, W. Antos and D.M. Lubman, Appl. Spectrosc., 44 (1990) 1391.

33 S.M. Michael, B.M. Chien and D.M. Lubman, 65 (1993) 2614.

34 B.M. Chien, S.M. Michael and D.M. Lubman, Anal. Chem., 65 (1993) 1916.

35 S.A. McLuckey, G.A. Glish, K.G. Asano and B.C. Grant, Anal. Chem., 60 (1988) 2220.

36 J.F.J. Todd and R.M. Waldren, Int. J. Mass Spectrom. Ion Phys., 29 (1979) 301.

37 C.S. O, H.A. Schuessler, Int. J. Mass Spectrom. Ion Phys., 40 (1981) 67.

38 C.S. O, H.A. Schuessler, Int. J. Mass Spectrom. Ion Phys., 40 (1981) 77.

39 C. Ma, S.M. Michael, M. Chien, J. Zhu and D.M. Lubman, Rev. Sci. Instrum., 63 (1992) 139.

40 J. G. Boyle and C.M. Whitehouse, Anal. Chem., 64 (1992) 2084.

41 R.G. Cooks and R.E. Kaiser Jr., Acc. Chem. Res., 23 (1990) 213.

42 R.G. Cooks, J. Williams, K. Cox, K. Kaiser and J. Schwartz, Rapid Commun. Mass Spectrom., 5 (1990) 327. 